\title{
A semilinear Black and Scholes partial differential equation for valuing American options: approximate solutions and convergence
}

\author{
F. E. BENTH $†$ K. H. KARLSEN ${ }^{\ddagger}$ AND K. REIKVAM ${ }^{\S}$ \\ Department of Mathematics, University of Oslo, \\ P.O. Box 1053, Blindern, N-0316 Oslo, Norway
}

[Received 28 April 2003 and in revised form 2 April 2004]

\begin{abstract}
In [7, we proved that the American (call/put) option valuation problem can be stated in terms of one single semilinear Black and Scholes partial differential equation set in a fixed domain. The semilinear Black and Scholes equation constitutes a starting point for designing and analyzing a variety of "easy to implement" numerical schemes for computing the value of an American option. To demonstrate this feature, we propose and analyze an upwind finite difference scheme of "predictor-corrector type" for the semilinear Black and Scholes equation. We prove that the approximate solutions generated by the predictor-corrector scheme respect the early exercise constraint and that they converge uniformly to the American option value. A numerical example is also presented. Besides the predictor-corrector schemes, other methods for constructing approximate solution sequences are discussed and analyzed.
\end{abstract}

Keywords: American option; semilinear Black and Scholes partial differential equation; viscosity solution; approximate solutions; numerical schemes; convergence.

\section{Contents}

\begin{tabular}{ll}
\hline 1. & Introduction \\
\hline
\end{tabular}

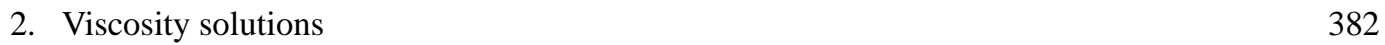

3. Semidiscrete approximations 384

\begin{tabular}{lr}
\hline 4. & The predictor-corrector scheme \\
\hline
\end{tabular}

\begin{tabular}{ll}
\hline 5. The Brennan and Schwartz algorithm & 397
\end{tabular}

\begin{tabular}{lll}
\hline 6. A numerical example & 399
\end{tabular}

\section{Introduction}

Let $T>0$ be fixed and $t<T$. Suppose that the price dynamics of a dividend paying stock $X_{S}$ is governed by a geometric Brownian motion (under the unique equivalent martingale measure $Q$ ), i.e., it evolves according to the stochastic differential equation

$$
d X(s)=(r-d) X(s) d s+\sigma X(s) d W(s), \quad s \in(t, T],
$$

where $d \geqslant 0$ is the constant dividend yield for the stock, $r \geqslant 0$ is the risk-free interest rate, $\sigma>0$ is the volatility, and $\{W(s) \mid s \in[0, T]\}$ is a standard Brownian motion. Starting at time $t$ with initial

\footnotetext{
${ }^{\dagger}$ Also Agder University College, Department of Economics and Business Administration, Serviceboks 422, N-4604 Kristiansand, Norway. Email: fredb@math.uio.no; URL: http://www.math.uio.no/fredb/

${ }^{\ddagger}$ Email: kennethk@math.uio.no; URL: http://www.math.uio.no/kennethk/

${ }^{\S}$ Email: kre@ math.uio.no; URL: http://www.math.uio.no/kre/
} 
condition $X(t)=x$, it is well known that the arbitrage-free value of an American option with expiration at time $T$ is given by

$$
V(t, x)=\sup _{t \leqslant \tau \leqslant T} \mathbb{E}^{t, x}\left[e^{-r(\tau-t)} g(X(\tau))\right],
$$

where the supremum is taken over all $\mathcal{F}_{t}$ stopping times $\tau \in[t, T], \mathbb{E}^{t, x}$ denotes expectation under the equivalent martingale measure conditioned on $X(t)=x$, and $g: \mathbb{R} \rightarrow \mathbb{R}$ is the payoff function. Herein we will focus on call and put options, i.e. options with payoff

$$
g(x)= \begin{cases}(x-K)^{+}, & \text {call option, } \\ (K-x)^{+}, & \text {put option, }\end{cases}
$$

where $K>0$ is the strike price. In this paper, we use Duffie [10], Karatzas and Shreve [17], Musiela and Rutkowski [19], and Myneni [20] as general references on the American option valuation problem.

In the literature one can find two main approaches to determining the function $V$ in 1.1: (i) the free boundary problem formulation and (ii) the quasi-variational inequality formulation. It is well known that there is no explicit formula for $V$, as opposed to the value of an European option for which an analytical formula exists. Consequently, with both approaches one has to resort to numerical schemes for finding $V$. However, the two approaches lend themselves to different numerical schemes. We refer to [10, 17, 19, 20] (and also the references therein) for mathematical and numerical aspects of the free boundary problem and quasi-variational inequality formulations as well as their advantages and disadvantages.

In [7], we presented a new approach to determining the value of an American option. The function $V$ in 1.1 is the value function of an optimal stopping problem for which the dynamic programming principle holds [24]. Using the dynamic programming principle, we proved in [7] that $V$ uniquely solves (in a viscosity solution sense) the following semilinear Black and Scholes equation set in a fixed domain:

$$
\partial_{t} v+(r-d) x \partial_{x} v+\frac{1}{2} \sigma^{2} x^{2} \partial_{x}^{2} v-r v=-q(x, v), \quad(t, x) \in Q_{T},
$$

where $Q_{T}$ denotes the time-space cylinder $[0, T) \times[0, \infty)$. The nonlinear reaction term

$$
q: \mathbb{R} \times \mathbb{R} \rightarrow[0, \infty)
$$

takes the form

$$
q(x, v)=c(x) H(g(x)-v),
$$

with $c: \mathbb{R} \rightarrow[0, \infty)$ being the "cash flow" function

$$
c(x)= \begin{cases}(d x-r K)^{+}, & \text {call option, } \\ (r K-d x)^{+}, & \text {put option, }\end{cases}
$$

and $H: \mathbb{R} \rightarrow[0, \infty)$ the Heaviside function

$$
H(\xi)= \begin{cases}0, & \xi<0 \\ 1, & \xi \geqslant 0 .\end{cases}
$$

We augment 1.3 with the terminal condition

$$
v(T, x)=g(x), \quad x \in[0, \infty),
$$


where $g$ is the payoff function in $(1.2)$. One should note that in $(1.3)$ there is no free boundary that needs to be computed as part of the solution nor are there "side constraints" that need to be satisfied by the solution (as opposed to the quasi-variational formulation). We refer to [7] for the motivation behind the semilinear Black and Scholes equation (1.3) and its rigorous derivation from (1.1) via the dynamic programming principle. We also refer to [7] for an overview of relevant literature. Here we only mention that (1.3) can be viewed as an infinitesimal (partial differential equation) version of the well known early exercise premium representation of the American option (i.e., the separation of the American option price into the corresponding European option price plus an early exercise premium) and that an initial motivation for our work in [7] was [18].

It was pointed out in [7] that the semilinear Black and Scholes equation does not make sense as it stands in (1.3) if classical (i.e., $C^{1,2}$ ) solutions-or more generally continuous viscosity solutions [9, 11] - are sought. This is related to the fact that the nonlinearity $v \mapsto q(x, v)$ in 1.3 is discontinuous. Guided by the dynamic programming principle, we suggested in [7] a suitable definition of a viscosity solution for (1.3). We recall this definition in Section 2 of the present paper. It was proved in [7] that the function $V$ defined in 1.1$]$ is the unique such viscosity solution of (1.3) satisfying the terminal condition (1.7). In other words, the terminal value problem (1.3)-1.7) constitutes an alternative formulation of the American (call/put) option valuation problem.

From a practical point of view, the advantage of the semilinear Black and Scholes equation (1.3) is that it suggests a natural recipe for constructing "easy to implement" numerical schemes for valuing American options. In fact, any European option solver can be turned into an American option solver via (1.3). In Section 4, we devise an upwind finite difference scheme of "predictorcorrector type" for 1.3. From (1.1), it is seen that the American option value $V$ always satisfies the early exercise constraint

$$
V \geqslant g \quad \text { on } \overline{Q_{T}}, \quad \overline{Q_{T}}:=[0, T] \times[0, \infty) .
$$

The suggested predictor-corrector scheme automatically satisfies a discrete analog of (1.8). Using the Barles-Ishii-Perthame weak viscosity limit method [9, 11], we give an easy $L_{\text {loc }}^{\infty}$ (i.e., uniform on compacta) convergence proof for the predictor-corrector scheme. A numerical example is presented in Section 6

We must stress that the particular numerical scheme studied herein is chosen just for its simplicity in terms of presentation, mathematical analysis, and implementation. But, at the same time, this simple choice illustrates the basic advantages of using the semilinear Black and Scholes equation (1.3) as the governing partial differential equation for American option valuation. In practical applications, however, one should use numerical schemes that result from a more sophisticated discretization of (1.3) than the one used herein.

In addition to the numerical scheme, we also analyze various sequences of "semidiscrete approximate solutions", which are obtained by solving "approximate semilinear Black and Scholes equations" in which the discontinuous right-hand side $q$ in 1.3 has been replaced by some continuous approximation. This is the topic of Section 3 . Related to this, let us mention that some of the so-called penalty schemes found in recent computational finance literature (see, e.g., [12, 21, 25]) can be interpreted as numerical schemes obtained by discretizing an approximate semilinear Black and Scholes equation. A consequence of this point of view is that one can adopt the techniques developed herein to give easy convergence proofs for penalty schemes. We make a further remark about this in Section 4

To demonstrate that the mathematical framework herein can be used to analyze other numerical schemes for valuing American options as well, we give in Section 5 a rather elementary convergence 
proof for a numerical scheme based on the classical Brennan and Schwartz algorithm [8] and the finite difference approach.

Finally, we mention that future work will be devoted to extending the semilinear Black and Scholes equation and its mathematical/numerical theory to the multi-asset setting.

\section{Viscosity solutions}

In this section, we recall from [7] the definition of a viscosity solution for the semilinear Black and Scholes equation. For a general introduction to the viscosity solution theory, we refer to Crandall, Ishii, and Lions [9] and Fleming and Soner [11]. We also recall from [9, 11] how to perform weak limit operations with viscosity solutions. This will be needed later when we prove convergence of various sequences of approximate solutions.

Before stating the definition of a viscosity solution, we need to introduce some notation. We start with the following spaces of semicontinuous functions:

$$
\begin{aligned}
& \operatorname{USC}\left(\overline{Q_{T}}\right)=\left\{v: \overline{Q_{T}} \rightarrow \mathbb{R} \cup\{-\infty\} \mid v \text { is upper semicontinuous }\right\}, \\
& \operatorname{LSC}\left(\overline{Q_{T}}\right)=\left\{v: \overline{Q_{T}} \rightarrow \mathbb{R} \cup\{+\infty\} \mid v \text { is lower semicontinuous }\right\} .
\end{aligned}
$$

Next, $C^{\alpha, \beta}\left(\overline{Q_{T}}\right)$ denotes the space of functions on $\overline{Q_{T}}$ that are $\alpha \geqslant 0$ times continuously differentiable in $t$ and $\beta \geqslant 0$ times continuously differentiable in $x$. The space of continuous functions on $\overline{Q_{T}}$ is denoted by $C\left(\overline{Q_{T}}\right)$, i.e., $C\left(\overline{Q_{T}}\right)=C^{0,0}\left(\overline{Q_{T}}\right)$.

In what follows, we will need the nonlinear functions $q^{*}, q_{*}: \mathbb{R} \times \mathbb{R} \rightarrow[0, \infty)$ defined by

$$
q^{*}(x, v)=c(x) H^{*}(g(x)-v), \quad q_{*}(x, v)=c(x) H_{*}(g(x)-v),
$$

where $H^{*}, H_{*}: \mathbb{R} \rightarrow[0, \infty)$ are defined as

$$
H^{*}(\xi)=\left\{\begin{array}{ll}
0, & \xi<0, \\
1, & \xi \geqslant 0,
\end{array} \quad H_{*}(\xi)= \begin{cases}0, & \xi \leqslant 0 \\
1, & \xi>0\end{cases}\right.
$$

REMARK Observe that $q^{*}$ and $q_{*}$ are respectively upper and lower semicontinuous on $\mathbb{R} \times \mathbb{R}$. Moreover, we have $q_{*} \leqslant q^{*}$ and $q^{*} \equiv q$. In fact, $q^{*}$ and $q_{*}$ are respectively the upper and lower semicontinuous envelopes of the nonlinear function $q$ defined in [1.6] (see [7]).

The fact that the mapping $v \mapsto q(x, v)$ is discontinuous makes it a nontrivial matter to decide what one should mean by a viscosity solution or even a classical $C^{1,2}$ solution of 1.3 . Using the dynamic programming principle in optimal stopping theory, we show in [7] that the notion of viscosity solution found in Definition 2.1 below is the natural one; that is, it identifies the American option value as the unique viscosity solution of the terminal value problem (1.3)-1.7). Remarkably, it turns out that this definition of a viscosity solution is an adaptation to our setting of the one used by Ishii [13] for first order Hamilton-Jacobi equations with discontinuous Hamiltonians.

DEFINITION $2.1 \quad$ (i) A locally bounded function $v \in \operatorname{USC}\left(\overline{Q_{T}}\right)$ is a viscosity subsolution of 1.3 if and only if for all $\phi \in C^{1,2}\left(\overline{Q_{T}}\right)$ we have:

$\left\{\begin{array}{l}\text { for each }(t, x) \in Q_{T} \text { being a local maximizer of } v-\phi, \\ \partial_{t} \phi(t, x)+(r-d) x \partial_{x} \phi(t, x)+\frac{1}{2} \sigma^{2} x^{2} \partial_{x}^{2} \phi(t, x)-r v(t, x)+q^{*}(x, v(t, x)) \geqslant 0 .\end{array}\right.$

If, in addition, $\left.v\right|_{t=T} \leqslant g$ on $[0, \infty)$, then $v$ is a viscosity subsolution of [1.3]-(1.7). 
(ii) A locally bounded function $v \in \operatorname{LSC}\left(\overline{Q_{T}}\right)$ is a (viscosity) supersolution of (1.3) if and only if for all $\phi \in C^{1,2}\left(\overline{Q_{T}}\right)$ we have:

$\left\{\begin{array}{l}\text { for each }(t, x) \in Q_{T} \text { being a local minimizer of } v-\phi, \\ \partial_{t} \phi(t, x)+(r-d) x \partial_{x} \phi(t, x)+\frac{1}{2} \sigma^{2} x^{2} \partial_{x}^{2} \phi(t, x)-r v(t, x)+q_{*}(x, v(t, x)) \leqslant 0 .\end{array}\right.$

If, in addition, $\left.v\right|_{t=T} \geqslant g$ on $[0, \infty)$, then $v$ is a viscosity supersolution of (1.3)-(1.7).

(iii) A function $v \in C\left(\overline{Q_{T}}\right)$ is a viscosity solution of $(1.3)$ if and only if it is simultaneously a viscosity sub- and supersolution of (1.3). If, in addition, $\left.v\right|_{t=T}=g$ on $[0, \infty)$, then $v$ is a viscosity solution of (1.3) 1.7 .

REMARK For convenience, we adopt the terms subsolution and supersolution instead of viscosity subsolution and viscosity supersolution. Furthermore, it is well known (see, e.g., [9, 11]) that we can replace "local" by "strict local" or "global" by "strict global". We can also assume that the extremum of $v-\phi$ has the value zero. There are equivalent formulations of sub- and supersolutions based on so-called semijets (or semidifferentials). These formulations were used in [7], but they will not be needed in this paper.

REMARK Note that if $v$ is a subsolution (supersolution) for $x>0$, then it is automatically a subsolution (supersolution) for $x \geqslant 0$. We refer to [7] for a proof of this result.

The remaining part of this section is devoted to weak (half-relaxed) limit operations with viscosity solutions [9, 11]. This will set the scene for the convergence proofs presented in the subsequent sections. Regarding limit operations with viscosity solutions, the following comparison principle will be of fundamental importance:

THEOREM 2.1 ([7]) Let $v_{\text {sub }}$ and $v_{\text {sup }}$ be respectively a sub- and supersolution of (1.3)-(1.7). Suppose there exists a finite constant $C$ such that

$$
v_{\text {sub }}(t, x),-v_{\text {sup }}(t, x) \leqslant C(1+x), \quad(t, x) \in \overline{Q_{T}} .
$$

Then

$$
v_{\text {sub }} \leqslant v_{\text {sup }} \quad \text { on } \overline{Q_{T}} .
$$

Later we shall repeatedly be faced with the problem of passing to the limit as $\varepsilon \downarrow 0$ in various sequences $v_{\varepsilon}: \overline{Q_{T}} \rightarrow \mathbb{R}$ of approximate solutions. A natural procedure for doing so is to prove that $v_{\varepsilon}$ is uniformly bounded (in $\varepsilon$ ) as well as equicontinuous on compacta. The Ascoli-Arzelà compactness theorem then gives $L_{\text {loc }}^{\infty}$ (i.e., uniform on compacta) convergence along a subsequence of $v_{\varepsilon}$ to a locally bounded and continuous function $v$. However, a merit of the viscosity solution theory is that one can dispense with the equicontinuity estimate provided there is a comparison principle in the class of semicontinuous sub- and supersolutions (Theorem 2.1). This limiting procedure is known as the Barles-Ishii-Perthame weak limit method [3, 14]. For completeness, we recall here the definitions of the upper and lower weak (or half-relaxed) limits of $v_{\varepsilon}$.

DEFINITION 2.2 Suppose $v_{\varepsilon}$ is locally uniformly bounded.

(i) The upper weak limit of $v_{\varepsilon}$, denoted by $\bar{v}$, is defined as

$$
\begin{aligned}
\bar{v}(t, x) & =\limsup _{\overline{Q_{T}} \ni(s, y) \rightarrow(t, x)} v_{\varepsilon}(s, y) \\
& =\lim _{\delta \downarrow 0} \sup _{\delta \downarrow}\left\{v_{\varepsilon}(s, y)\left|(s, y) \in \overline{Q_{T}},\right| t-s|,| x-y \mid \leqslant \delta, 0<\varepsilon \leqslant \delta\right\} .
\end{aligned}
$$


(ii) The lower weak limit of $v_{\varepsilon}$, denoted by $\underline{v}$, is defined as

$$
\begin{aligned}
\underline{v}(t, x) & =\liminf _{\overline{Q_{T}} \ni(s, y) \rightarrow(t, x)} v_{\mathcal{E}}(s, y) \\
& =\liminf _{\delta \downarrow 0}\left\{v_{\varepsilon}(s, y)\left|(s, y) \in \overline{Q_{T}},\right| t-s|,| x-y \mid \leqslant \delta, 0<\varepsilon \leqslant \delta\right\} .
\end{aligned}
$$

Since $v_{\varepsilon}$ is locally uniformly bounded, the weak limits $\bar{v}$ and $\underline{v}$ are well defined (finite). Some properties (to be used later) of the weak limits are given in the next lemma (whose proof can found in, e.g., [9, 11]]).

LEMMA 2.1 (i) The upper weak limit $\bar{v}$ belongs to $\operatorname{USC}\left(\overline{Q_{T}}\right)$ and the lower weak limit $\underline{v}$ belongs to $\operatorname{LSC}\left(\overline{Q_{T}}\right)$.

(ii) If $\bar{v}=\underline{v}=v$ on a compact subset of $\overline{Q_{T}}$, then $v$ is continuous and $v_{\varepsilon} \rightarrow v$ in $L^{\infty}$ (i.e., uniformly) on this set as $\varepsilon \downarrow 0$.

(iii) Let $v_{\varepsilon} \in \operatorname{USC}\left(\overline{Q_{T}}\right)$ (resp. $\operatorname{LSC}\left(\overline{Q_{T}}\right)$ ) be locally uniformly (in $\varepsilon$ ) bounded. Let $(t, x) \in \overline{Q_{T}}$ be a strict local maximizer of $\bar{v}-\phi$ (resp. minimizer for $\underline{v}-\phi), \phi \in C^{1,2}\left(\overline{Q_{T}}\right)$. Then there exist subsequences, which we do not relabel, $\left(t_{\varepsilon}, x_{\varepsilon}\right) \rightarrow(t, x)$ and $v_{\varepsilon}\left(t_{\varepsilon}, x_{\varepsilon}\right) \rightarrow \bar{v}(t, x)$ (resp. $\underline{v}(t, x))$ as $\varepsilon \downarrow 0$ such that $\left(t_{\varepsilon}, x_{\varepsilon}\right)$ is a local maximizer (resp. minimizer) of $v_{\varepsilon}-\phi$ for each $\varepsilon>0$.

Regarding the general use of the weak limit method as a tool for proving convergence of approximate solutions of fully nonlinear degenerate second order partial differential equations, we refer to [4, 9, 11] and the references therein. For some concrete applications to partial differential equations arising in finance theory, see [1, 2] and the references therein.

\section{Semidiscrete approximations}

In this section we present and analyze several examples of "semidiscrete" approximate solutions of the semilinear Black and Scholes equation (1.3). These approximations can be used to construct "fully discrete" numerical schemes for computing the value of an American option (see, e.g., [12, 21, 25] as well as Section 5 herein). We also introduce the basic techniques for analyzing approximate solution sequences associated with the semilinear Black and Scholes equation.

Let us start with the classical penalization technique [5, 6], which considers the following equation for each $\varepsilon>0$ :

$$
\partial_{t} v_{\varepsilon}+(r-d) x \partial_{x} v_{\varepsilon}+\frac{1}{2} \sigma^{2} x^{2} \partial_{x}^{2} v_{\varepsilon}-r v_{\varepsilon}=-\frac{1}{\varepsilon}\left(g(x)-v_{\varepsilon}\right)^{+}, \quad(t, x) \in Q_{T},
$$

with terminal data

$$
v_{\varepsilon}(T, x)=g(x), \quad x \in[0, \infty)
$$

From standard viscosity solution theory [9, 11], we know that there exists a unique viscosity solution $v_{\varepsilon}$ to 3.1 - 3.2 satisfying $0 \leqslant v_{\varepsilon} \leqslant C(1+x)$ on $\overline{Q_{T}}$, where the constant $C$ is independent of $\varepsilon$. Regarding the penalization method, we have the following theorem:

THEOREM 3.1 Let $v$ be the unique viscosity solution of $(1.3-1.7)$. For each $\varepsilon>0$, let $v_{\varepsilon}$ be the unique viscosity solution of 3.1]-3.2. Then $v_{\varepsilon} \rightarrow v$ in $L_{\mathrm{loc}}^{\infty}\left(Q_{T}\right)$ as $\varepsilon \downarrow 0$. 
Proof. Let $\bar{v}$ and $\underline{v}$ be the upper and lower weak limits of $v_{\varepsilon}$ (see Definition 2.2. From Lemma2.1. $\bar{v} \in \operatorname{USC}\left(\overline{Q_{T}}\right)$ and $\underline{v} \in \operatorname{LSC}\left(\overline{Q_{T}}\right)$. Obviously, $0 \leqslant \bar{v}, \underline{v} \leqslant C(1+x)$ on $\overline{Q_{T}}$.

We prove first that $\underline{v}$ is a supersolution of $(1.3)-(1.7)$, starting with the terminal condition (1.7). To get a contradiction, suppose there exist $y \in[0, \infty)$ and $\delta>0$ such that $\underline{v}(T, y) \leqslant g(y)-\delta$. Pick sequences $\left(t_{\varepsilon}, x_{\varepsilon}\right) \rightarrow(T, y)$ and $v_{\varepsilon}\left(t_{\varepsilon}, x_{\varepsilon}\right) \rightarrow \underline{v}(T, y)$ as $\varepsilon \downarrow 0$. Because of [3.2], $t_{\varepsilon}<T$ for all $\varepsilon \leqslant \varepsilon_{0}$ and some $\varepsilon_{0}>0$. We next pick a function $\tilde{g} \in C^{2}([0, \infty))$ such that $\tilde{g} \leqslant g$ on $[0, \infty)$, $\tilde{g}(y)=g(y)-\delta / 2$, and $\tilde{g}=$ const on $[\tilde{K}, \infty)$ with $\tilde{K}>y$. Define the function $G=-C(T-t)+\tilde{g}$ for a constant $C>0$. Observe that $G<g$ on $Q_{T}$. For an appropriate choice of $C, G$ turns out to be a subsolution of $3.1-(3.2)$ :

$$
\begin{aligned}
\partial_{t} G(t, x)+(r-d) x \partial_{x} G(t, x)+\frac{1}{2} \sigma^{2} x^{2} \partial_{x}^{2} G(t, x)-r G(t, x)+\frac{1}{\varepsilon}(g(x)-G)^{+} \\
\\
\geqslant C+(r-d) x \partial_{x} \tilde{g}(x)+\frac{1}{2} \sigma^{2} x^{2} \partial_{x}^{2} \tilde{g}(x)-r \tilde{g}(x) .
\end{aligned}
$$

By choosing

$$
C \geqslant-\min _{x \in[0, \infty)}\left\{(r-d) x \partial_{x} \tilde{g}(x)+\frac{1}{2} \sigma^{2} x^{2} \partial_{x}^{2} \tilde{g}(x)-r \tilde{g}(x)\right\},
$$

we see that $G$ becomes a subsolution. Observe that the minimum is finite since $\tilde{g}=$ const on $[\tilde{K}, \infty)$. The comparison principle for $\sqrt{3.1}-(3.2)$ (see [9, 11]) implies $v_{\varepsilon} \geqslant G$ on $\overline{Q_{T}}$ for any $\varepsilon \in\left(0, \varepsilon_{0}\right]$. Letting $\varepsilon \downarrow 0$, we get $\underline{v} \geqslant G$ on $Q_{T}$ and, in particular, $\underline{v}(T, y) \geqslant \tilde{g}(y)=g(y)-\delta / 2$, which is a contradiction. This concludes the proof of the terminal condition $\left.\underline{v}\right|_{t=T} \geqslant g$ on $[0, \infty)$. Next we prove that $\underline{v}$ is a supersolution of $(1.3)$. Let $(t, x) \in Q_{T}$ be a strict local minimizer of $\underline{v}-\phi$, $\phi \in C^{1,2}\left(\overline{Q_{T}}\right)$. First, we claim that

$$
\underline{v}(t, x) \geqslant g(x) .
$$

To get a contradiction, suppose $\underline{v}(t, x) \leqslant g(x)-\delta$ for some $\delta>0$. By Lemma 2.1, there exist sequences $\left(t_{\varepsilon}, x_{\varepsilon}\right) \rightarrow(t, x)$ and $v_{\varepsilon}\left(t_{\varepsilon}, x_{\varepsilon}\right) \rightarrow \underline{v}(t, x)$ as $\varepsilon \downarrow 0$ such that $\left(t_{\varepsilon}, x_{\varepsilon}\right)$ is a local minimizer of $v_{\varepsilon}-\phi$ for each $\varepsilon$. Obviously, there exists an $\varepsilon(\delta)>0$ such that

$$
v_{\varepsilon}\left(t_{\varepsilon}, x_{\varepsilon}\right) \leqslant g\left(x_{\varepsilon}\right)-\delta / 2, \quad \forall \varepsilon \leqslant \varepsilon(\delta) .
$$

In view of this and since $v_{\varepsilon}$ is a supersolution of [3.1], we have (for $\varepsilon$ small enough)

$$
\begin{aligned}
\partial_{t} \phi\left(t_{\varepsilon}, x_{\varepsilon}\right)+(r-d) x_{\varepsilon} \partial_{x} \phi\left(t_{\varepsilon}, x_{\varepsilon}\right)+\frac{1}{2} \sigma^{2} x_{\varepsilon}^{2} \partial_{x}^{2} \phi\left(t_{\varepsilon}, x_{\varepsilon}\right) & -r v_{\varepsilon}\left(t_{\varepsilon}, x_{\varepsilon}\right) \\
& \leqslant-\frac{1}{\varepsilon}\left(g\left(x_{\varepsilon}\right)-v_{\varepsilon}\left(t_{\varepsilon}, x_{\varepsilon}\right)\right) \leqslant-\frac{\delta}{2 \varepsilon} .
\end{aligned}
$$

Letting $\varepsilon \downarrow 0$ in this inequality gives a contradiction since the left-hand side converges to a finite number while the right-hand side converges to $-\infty$. This proves 3.3 , which in turn implies $q_{*}(x, \underline{v}(t, x))=0$. To conclude the proof of the supersolution property of $\underline{v}$ at $(t, x)$, it only remains to let $\bar{\downarrow} \downarrow 0$ in the inequality

$$
\partial_{t} \phi\left(t_{\varepsilon}, x_{\varepsilon}\right)+(r-d) x_{\varepsilon} \partial_{x} \phi\left(t_{\varepsilon}, x_{\varepsilon}\right)+\frac{1}{2} \sigma^{2} x_{\varepsilon}^{2} \partial_{x}^{2} \phi\left(t_{\varepsilon}, x_{\varepsilon}\right)-r v_{\varepsilon}\left(t_{\varepsilon}, x_{\varepsilon}\right) \leqslant 0 .
$$

In [7], it is proved that $g$ is a subsolution of (1.3)-(1.7). We have just proved that $\underline{v}$ is a supersolution of (1.3)-1.7). Consequently, Theorem 2.1 tells us that

$$
\bar{v}, \underline{v} \geqslant g \quad \text { on } \overline{Q_{T}} .
$$


We prove next that $\bar{v}$ is a subsolution of (1.3)-(1.7), starting with the terminal condition (1.7). To get a contradiction, suppose there exist $y \in[0, \infty)$ and $\delta>0$ such that $\bar{v}(T, y) \geqslant g(y)+\delta$. Pick sequences $\left(t_{\varepsilon}, x_{\varepsilon}\right) \rightarrow(T, y)$ and $v_{\varepsilon}\left(t_{\varepsilon}, x_{\varepsilon}\right) \rightarrow \bar{v}(T, y)$ as $\varepsilon \downarrow 0$. Because of (3.2), $t_{\varepsilon}<T$ for all $\varepsilon \leqslant \varepsilon_{0}$ and some $\varepsilon_{0}>0$. We next pick a function $\tilde{g} \in C^{2}([0, \infty))$ such that $\tilde{g} \geqslant g$ on $[0, \infty), \tilde{g}(y)=g(y)+\delta / 2$, and $\tilde{g}=g$ on $[\tilde{K}, \infty)$ with $\tilde{K}>\max (y, K)$. Define the function $G=C(T-t)+\tilde{g}$ and note that $G \geqslant g$ on $\overline{Q_{T}}$. Following the same calculation as in the subsolution case, we see that if we choose

$$
C \geqslant \max _{x \in[0, \infty)}\left\{(r-d) x \partial_{x} \tilde{g}(x)+\frac{1}{2} \sigma^{2} x^{2} \partial_{x}^{2} \tilde{g}(x)-r \tilde{g}(x)\right\},
$$

then $G$ becomes a supersolution of (3.1)- 3.2). Note that the maximum is finite: For $x>\tilde{K}$ we have $\tilde{g}=g$. Thus, for a call option, $\tilde{g}(x)=x-K$ for $x>\tilde{K}$, and the expression inside the curly brackets is equal to $-d x+r K \leqslant-d \tilde{K}+r k$. For a put option, on the other hand, $\tilde{g}(x)=0$ for $x>\tilde{K}$. The comparison principle for [3.1)- 3.2) (see [9, 11]) then implies $v_{\varepsilon} \leqslant G$ on $\overline{Q_{T}}$ for any $\varepsilon \in\left(0, \varepsilon_{0}\right]$. Letting $\varepsilon \downarrow 0$, we get $\bar{v} \leqslant G$ on $\overline{Q_{T}}$ and thus $\bar{v}(T, y) \leqslant \tilde{g}(y)=g(y)+\delta / 2$, which is a contradiction. Hence $\left.\bar{v}\right|_{t=T} \leqslant g$ on $[0, \infty)$. Let us now prove that $\bar{v}$ is a subsolution of (1.3). Let $(t, x) \in Q_{T}$ be a strict local maximizer of $\bar{v}-\phi, \phi \in C^{1,2}\left(\overline{Q_{T}}\right)$. We use again Lemma 2.1 to find sequences $\left(t_{\varepsilon}, x_{\varepsilon}\right) \rightarrow(t, x)$ and $v_{\varepsilon}\left(t_{\varepsilon}, x_{\varepsilon}\right) \rightarrow \bar{v}(t, x)$ as $\varepsilon \downarrow 0$ such that $\left(t_{\varepsilon}, x_{\varepsilon}\right)$ is a local maximizer of $v_{\varepsilon}-\phi$ for each $\varepsilon$. If $\bar{v}(t, x)=g(x)$, then we use (3.4) and argue as follows: Since $\bar{v}(t, x)=g(x)$ and $\bar{v} \geqslant g$ on $\overline{Q_{T}}$, we conclude that $g-\phi$ has a local maximum at $(t, x)$. Since $g$ is a subsolution of (1.3) (see [7]), 2.2 follows. Finally, if $\bar{v}(t, x)>g(x)$, then it is clear that $v_{\varepsilon}\left(t_{\varepsilon}, x_{\varepsilon}\right)>g\left(x_{\varepsilon}\right)$ for any $\varepsilon$ sufficiently small. Since $v_{\varepsilon}$ is a subsolution of [3.1, we have (for any $\varepsilon$ small enough)

$$
\partial_{t} \phi\left(t_{\varepsilon}, x_{\varepsilon}\right)+(r-d) x_{\varepsilon} \partial_{x} \phi\left(t_{\varepsilon}, x_{\varepsilon}\right)+\frac{1}{2} \sigma^{2} x_{\varepsilon}^{2} \partial_{x}^{2} \phi\left(t_{\varepsilon}, x_{\varepsilon}\right)-r v_{\varepsilon}\left(t_{\varepsilon}, x_{\varepsilon}\right) \geqslant 0 .
$$

Since $\bar{v}(t, x)>g(x), q_{*}(x, \bar{v}(t, x))=0$. Letting $\varepsilon \downarrow 0$ in the above inequality thus yields the subsolution property of $\bar{v}$ at $(t, x)$.

Theorem 2.1 implies that $\bar{v} \leqslant \underline{v}$, and thus $\bar{v}=\underline{v}=v$. Lemma 2.1 then concludes the proof of the theorem.

Let $q_{\varepsilon}: \mathbb{R} \times \mathbb{R} \rightarrow \mathbb{R}$ designate a reasonable approximation to $q$. The term "reasonable" will be made precise through conditions (3.12) and 3.13 in Lemma 3.1 below. Another way to construct semidiscrete approximate solutions to the semilinear Black and Scholes equation (1.3) is to consider the following equation for each $\varepsilon>0$ :

$$
\partial_{t} v_{\varepsilon}+(r-d) x \partial_{x} v_{\varepsilon}+\frac{1}{2} \sigma^{2} x^{2} \partial_{x}^{2} v_{\varepsilon}-r v_{\varepsilon}+q_{\varepsilon}\left(x, v_{\varepsilon}\right)=0, \quad(x, t) \in Q_{T},
$$

which is augmented with terminal data

$$
v_{\varepsilon}(T, x)=g(x), \quad x \in[0, \infty) .
$$

Let us look at some choices of $q_{\varepsilon}$ of the form

$$
q_{\varepsilon}(x, v)=c(x) H_{\varepsilon}(g(x)-v), \quad \varepsilon>0,
$$

where $H_{\varepsilon}: \mathbb{R} \rightarrow \mathbb{R}$ is an approximation to the Heaviside function (1.6). One example may be the "symmetric approximation" 


$$
H_{\varepsilon}(\xi)= \begin{cases}0, & \xi<-\varepsilon, \\ (\xi+\varepsilon) / 2 \varepsilon, & -\varepsilon \leqslant \xi<\varepsilon, \\ 1 & \xi \geqslant \varepsilon .\end{cases}
$$

Similarly, we have the "approximation from above"

$$
H_{\varepsilon}(\xi)= \begin{cases}0, & \xi<-\varepsilon, \\ (\xi+\varepsilon) / \varepsilon, & -\varepsilon \leqslant \xi<0, \\ 1 & \xi \geqslant 0,\end{cases}
$$

as well as the "approximation from below"

$$
H_{\varepsilon}(\xi)= \begin{cases}0, & \xi<0 \\ \xi / \varepsilon, & 0 \leqslant \xi<\varepsilon \\ 1 & \xi \geqslant \varepsilon\end{cases}
$$

Our final example is (there are of course infinitely many more and they do not need to be smooth as is the case with the examples here)

$$
H_{\varepsilon}(\xi)=\frac{\varepsilon}{\varepsilon-\xi^{-}} .
$$

From standard viscosity solution theory [9, 11], we know that for any reasonable $q_{\varepsilon}$ there exists a unique viscosity solution $v_{\varepsilon}$ of (3.1)- 3.2) satisfying $0 \leqslant v_{\varepsilon} \leqslant C(1+x)$ on $\overline{Q_{T}}$, where the constant $C$ is independent of $\varepsilon$. The next lemma shows that viscosity solutions (in the sense of Definition 2.1) are stable with respect to weak limits.

LEMMA 3.1 For each $\varepsilon>0$, let $q_{\varepsilon}$ be a locally uniformly bounded function such that the comparison principle holds for $(3.5)-(3.6)$, and $v_{\varepsilon} \in \operatorname{USC}\left(\overline{Q_{T}}\right)$ (resp. $\operatorname{LSC}\left(\overline{Q_{T}}\right)$ ) be a locally uniformly bounded subsolution (resp. supersolution) of (3.5)-(3.6). Suppose there exist $\varepsilon_{0}>0$ and finite constants $\underline{C}, \bar{C} \geqslant 0$ such that

$$
\begin{array}{ll}
q_{\varepsilon}(x, v) \leqslant c(x)+\bar{C} & \text { whenever } g(x)-v<0 \text { and } \varepsilon \leqslant \varepsilon_{0}, \\
q_{\varepsilon}(x, v) \geqslant c(x)-\underline{C} & \text { whenever } g(x)-v>0 \text { and } \varepsilon \leqslant \varepsilon_{0},
\end{array}
$$

where the "cash flow" function $c$ is defined in 1.5 . Let $\bar{q}$ and $q$ be respectively the upper and lower weak limits of $q_{\varepsilon}$. If

$$
\bar{q}(x, v) \leqslant q^{*}(x, v) \quad \forall(x, v) \in \mathbb{R} \times \mathbb{R}, \quad \underline{q}(x, v) \geqslant q_{*}(x, v) \quad \forall(x, v) \in \mathbb{R} \times \mathbb{R},
$$

where $q^{*}, q_{*}$ are defined in $\left(2.1\right.$, then the upper weak limit $\bar{v}$ (resp. lower weak limit $\underline{v}$ ) of $v_{\varepsilon}$ is a subsolution (resp. supersolution) of (1.3)-(1.7).

Proof. From Lemma 2.1, $\bar{v}$ is upper semicontinuous and $\underline{v}$ is lower semicontinuous. Also, $0 \leqslant$ $\bar{v}, \underline{v} \leqslant C(1+x)$ on $\overline{Q_{T}}$. Let us now prove that $\bar{v}$ satisfies the terminal condition. To this end, choose a function $\tilde{g} \in C^{2}([0, \infty))$ such that $\tilde{g} \geqslant g$ on $[0, \infty),\|\tilde{g}-g\|_{L^{\infty}([0, \infty))} \leqslant \delta$ for some $\delta>0$, and $\tilde{g}=g$ on $[\tilde{K}, \infty)$ with $\tilde{K}$ sufficiently large. Define the function $G=C(T-t)+\tilde{g}$ for a constant 
$C>0$, and note that $G>g$ on $Q_{T}$. Observe that, for any $\varepsilon \leqslant \varepsilon_{0}$ and $(t, x) \in Q_{T}$,

$$
\begin{gathered}
\partial_{t} G(t, x)+(r-d) x \partial_{x} G(t, x)+\frac{1}{2} \sigma^{2} x^{2} \partial_{x}^{2} G(t, x)-r G(t, x)+q_{\varepsilon}(x, G(t, x)) \\
\leqslant-C+(r-d) x \partial_{x} \tilde{g}(x)+\frac{1}{2} \sigma^{2} x^{2} \partial_{x}^{2} \tilde{g}(x)-r \tilde{g}(x)+q_{\varepsilon}(x, G(t, x)) \\
\quad \leqslant-C+(r-d) x \partial_{x} \tilde{g}(x)+\frac{1}{2} \sigma^{2} x^{2} \partial_{x}^{2} \tilde{g}(x)-r \tilde{g}(x)+c(x)+\bar{C},
\end{gathered}
$$

where we have used (3.12) to derive the second inequality. Now choose

$$
C \geqslant \bar{C}+\max _{x \in[0, \infty)}\left\{(r-d) x \partial_{x} \tilde{g}(x)+\frac{1}{2} \sigma^{2} x^{2} \partial_{x}^{2} \tilde{g}(x)-r \tilde{g}(x)+c(x)\right\} .
$$

Note that the maximum is finite since

$$
(r-d) x \partial_{x} \tilde{g}(x)+\frac{1}{2} \sigma^{2} x^{2} \partial_{x}^{2} \tilde{g}(x)-r \tilde{g}(x)=-c(x), \quad x>\tilde{K} .
$$

Plugging this $C$ into (3.14), we conclude that $G$ is a supersolution of (3.5) and obviously also of (3.5)-3.6 (at least when $\varepsilon \leqslant \varepsilon_{0}$ ). The comparison principle for 3.5]-3.6. (see [9, 11]) then implies $v_{\varepsilon} \leqslant G$ on $\overline{Q_{T}}$ for any $\varepsilon \leqslant \varepsilon_{0}$. Hence $\bar{v} \leqslant G$ on $[0, \infty)$ and, in particular, $\bar{v}(T, x) \leqslant$ $\tilde{g}(x) \leqslant g(x)+\delta$ for $x \in[0, \infty)$. Since $\delta>0$ was arbitrary, we conclude that $\left.\bar{v}\right|_{t=T} \leqslant g$ on $[0, \infty)$. Similarly, we can prove that $\underline{v}$ satisfies the terminal condition. This time we choose a function $\tilde{g} \in C^{2}([0, \infty))$ such that $\tilde{g} \leqslant g$ on $[0, \infty),\|\tilde{g}-g\|_{L^{\infty}([0, \infty))} \leqslant \delta$, and, for a sufficiently large $\tilde{K}$, $\tilde{g}=g$ on $[\tilde{K}, \infty)$. Define the function $G=-C(T-t)+\tilde{g}$ for a constant $C>0$, and note that $G<g$ on $Q_{T}$. In view of 3.12, it is not difficult to see that if we choose $C$ so that

$$
C \geqslant \underline{C}-\min _{x \in[0, \infty)}\left\{(r-d) x \partial_{x} \tilde{g}(x)+\frac{1}{2} \sigma^{2} x^{2} \partial_{x}^{2} \tilde{g}(x)-r \tilde{g}(x)+c(x)\right\},
$$

then $G$ becomes a subsolution of 3.1)-3.2 whenever $\varepsilon \leqslant \varepsilon_{0}$. The comparison principle for 3.5 (3.6) (see [9, 11]) then implies $v_{\varepsilon} \geqslant G$ on $Q_{T}$ (for small enough $\varepsilon$ ). We now end the proof as we $\operatorname{did}$ for $\bar{v}$ and obtain $\left.\underline{v}\right|_{t=T} \geqslant g$ on $[0, \infty)$.

Next we prove that $\bar{v}, \underline{v}$ are respectively sub- and supersolutions of $(1.3)$. We present here only the subsolution case (the supersolution case is similar). Let $(t, x) \in Q_{T}$ be a strict local maximizer of $\bar{v}-\phi, \phi \in C^{1,2}\left(\overline{Q_{T}}\right)$. By Lemma 2.1. there exist sequences $\left(t_{\varepsilon}, x_{\varepsilon}\right) \rightarrow(t, x)$ and $v_{\varepsilon}\left(t_{\varepsilon}, x_{\varepsilon}\right) \rightarrow$ $\bar{v}(t, x)$ as $\varepsilon \downarrow 0$ such that each $\left(t_{\varepsilon}, x_{\varepsilon}\right)$ is a local maximizer of $v_{\varepsilon}-\phi$, and hence

$$
\begin{aligned}
\partial_{t} \phi\left(t_{\varepsilon}, x_{\varepsilon}\right)+(r-d) x_{\varepsilon} \partial_{x} \phi\left(t_{\varepsilon}, x_{\varepsilon}\right)+\frac{1}{2} \sigma^{2} x_{\varepsilon}^{2} \partial_{x}^{2} \phi\left(t_{\varepsilon}, x_{\varepsilon}\right)- & r v_{\varepsilon}\left(t_{\varepsilon}, x_{\varepsilon}\right) \\
& +q_{\varepsilon}\left(x_{\varepsilon}, v_{\varepsilon}\left(t_{\varepsilon}, x_{\varepsilon}\right)\right) \geqslant 0,
\end{aligned}
$$

or, after rearranging,

$$
\begin{aligned}
q_{\varepsilon}\left(x_{\varepsilon}, v_{\varepsilon}\left(t_{\varepsilon}, x_{\varepsilon}\right)\right) \geqslant & -\partial_{t} \phi\left(t_{\varepsilon}, x_{\varepsilon}\right)-(r-d) x_{\varepsilon} \partial_{x} \phi\left(t_{\varepsilon}, x_{\varepsilon}\right) \\
& -\frac{1}{2} \sigma^{2} x_{\varepsilon}^{2} \partial_{x}^{2} \phi\left(t_{\varepsilon}, x_{\varepsilon}\right)+r v_{\varepsilon}\left(t_{\varepsilon}, x_{\varepsilon}\right) .
\end{aligned}
$$


Obviously, by (3.13) and the definition of $\bar{q}$, we have

$$
q^{*}(x, \bar{v}(t, x)) \geqslant \bar{q}(x, \bar{v}(t, x)) \geqslant \limsup _{\varepsilon \downarrow 0} q_{\varepsilon}\left(x_{\varepsilon}, v_{\varepsilon}\left(t_{\varepsilon}, x_{\varepsilon}\right)\right) .
$$

Hence, taking lim sup on both sides in 3.16) and observing that lim sup coincides with lim on the right-hand side, we have the subsolution property of $\bar{v}$ at $(t, x)$ :

$$
\partial_{t} \phi(t, x)+(r-d) x \partial_{x} \phi(t, x)+\frac{1}{2} \sigma^{2} x^{2} \partial_{x}^{2} \phi(t, x)-r \bar{v}(t, x)+q^{*}(x, \bar{v}(t, x)) \geqslant 0 .
$$

A consequence of the previous lemma is the following theorem:

THEOREM 3.2 For each $\varepsilon>0$, suppose $q_{\varepsilon}$ is a locally uniformly bounded function such that the comparison principle holds for (3.5)-(3.6), (3.12) and (3.13) hold, and $v_{\varepsilon}$ is a locally uniformly bounded viscosity solution of 3.5-3.6. Then $v_{\varepsilon} \rightarrow v$ as $\varepsilon \downarrow 0$, where $v$ is the unique viscosity solution of (1.3)-(1.7). The convergence takes place in $L_{\mathrm{loc}}^{\infty}\left(\overline{Q_{T}}\right)$.

Proof. By definition, $\underline{v} \leqslant \bar{v}$ on $\overline{Q_{T}}$. From Lemma 3.1. $\bar{v}$ and $\underline{v}$ are respectively sub- and supersolutions of (1.3)- (1.7) and $\bar{v}$ is a supersolution of (1.3)-(1.7). Theorem 2.1 yields therefore $\bar{v} \leqslant \underline{v}$ on $\overline{Q_{T}}$, and hence $\underline{v}=\bar{v}=v$ is the viscosity solution of $(1.3)$. The uniform convergence follows from Lemma 2.1

Regarding the choices of $q_{\varepsilon}$ discussed above, we have the following convergence theorem:

THEOREM 3.3 For each $\varepsilon>0$, let $v_{\varepsilon}$ be the unique viscosity solution of (3.5)- 3.6 with $q_{\varepsilon}$ defined via (3.7) and one of the choices (3.8), (3.9), (3.10), or (3.11). As $\varepsilon \downarrow 0, v_{\varepsilon}$ converges in $L_{\text {loc }}^{\infty}\left(\overline{Q_{T}}\right)$ to the unique viscosity solution $v$ of $[1.3-1.7]$.

Proof. In what follows, let $q_{\varepsilon}$ be any one of the choices mentioned in the theorem. Standard viscosity solution theory [9, 11] provides us with the existence of a unique viscosity solution of (3.5)-3.6) satisfying $0 \leqslant v_{\varepsilon} \leqslant C(1+x)$ on $\overline{Q_{T}}$, where $C$ is independent of $\varepsilon$. Moreover, the comparison principle holds. It is easy to check that $q_{\varepsilon}$ satisfies (3.12) and that the upper and lower weak limits $\bar{q}$ of and $\underline{q}$ of $q_{\varepsilon}$ coincide with $q^{*}$ and $q_{*}$ respectively. Hence an application of Theorem 3.2 concludes the proof.

\section{The predictor-corrector scheme}

The semilinear Black and Scholes equation (1.3) provides a natural recipe for turning any numerical scheme for the European option valuation problem into a numerical scheme for the American option valuation problem. We have chosen to illustrate this feature by devising a very simple explicit upwind finite difference scheme of "predictor-corrector type" for (1.3). It will become apparent, however, that everything presented in this section can be extended to other (more sophisticated) numerical schemes for $(1.3)$.

To implement a numerical scheme on a computer, we must truncate the infinite domain $[0, \infty)$ to a finite domain $[0, L)$, where $L<\infty$ is fixed, and then provide a reasonable boundary condition at $x=L$ (see (4.7) below). The truncation technique is a classical one in numerical finance (see [2] for a theoretical investigation of it). The choice of boundary condition at $x=L$ will not affect the theoretical convergence analysis given later. The reason is that we will let $L \uparrow \infty$ as $\Delta x \downarrow 0$ in the convergence analysis. 
Let $\Delta x>0$ and $\Delta t>0$ be the spatial and temporal discretization parameters, respectively. The spatial domain $[0, L]$ is then discretized into grid cells

$$
I_{j}=\left[x_{j-1 / 2}, x_{j+1 / 2}\right), \quad j=1, \ldots, J_{L}-1,
$$

where $x_{\ell}=\ell \Delta x$ for $\ell=0,1 / 2,1, \ldots, J_{L}-1, J_{L}-1 / 2, J_{L}$. Furthermore, we set $I_{0}=\left[0, x_{1 / 2}\right)$ and $I_{J_{L}}=\left[x_{J_{L}-1 / 2}, x_{J_{L}}\right]$. Choose the integer $J_{L}$ such that $J_{L} \Delta x=L$. For the convergence analysis, we let

$$
J_{L} \Delta x=L \uparrow \infty \quad \text { as } \Delta x \downarrow 0 .
$$

Similarly, the time interval $[0, T]$ is discretized into time strips

$$
I^{n}=\left[t^{n}, t^{n+1}\right), \quad n=N-1, \ldots, 0,
$$

where $t^{n}=n \Delta t$ for $n=0, \ldots, N$. The integer $N$ is chosen such that $N \Delta t=T$. We denote by $R_{j}^{n}$ the rectangle $I^{n} \times I_{j}$. For $j=0, \ldots, J_{L}$ and $n=N, N-1, \ldots, 0, V_{j}^{n}$ denotes the predictorcorrector approximation (yet to be defined) associated with the point $\left(t^{n}, x_{j}\right)$.

We extend the difference solution $\left\{V_{j}^{n}\right\}$ to all of $Q_{T}^{L}=[0, T] \times[0, L]$ by setting

$$
v_{\Delta}(t, x)= \begin{cases}V_{j}^{n}, & (t, x) \in R_{j}^{n}, \quad j=0, \ldots, J_{L}, n=N-1, \ldots, 0, \\ V_{j}^{N}, & t=T, x \in I_{j}, \quad j=0, \ldots, J_{L},\end{cases}
$$

where $\Delta$ is used as short-hand notation for $\Delta x$. Let us now introduce the explicit predictor-corrector scheme. To this end, set $\lambda=\Delta t / \Delta x$ and $\mu=\Delta t /(\Delta x)^{2}$. To simplify the presentation, we use $\Delta_{+}$and $\Delta_{-}$to designate the difference operators in the $x$ direction:

$$
\Delta_{+} V_{j}^{n}=V_{j+1}^{n}-V_{j}^{n}, \quad \Delta_{-} V_{j}^{n}=V_{j}^{n}-V_{j-1}^{n} .
$$

For the same reason, we introduce the upwind numerical flux function $F: \mathbb{R} \times \mathbb{R} \rightarrow \mathbb{R}$ defined by

$$
F(a, b)= \begin{cases}b & \text { when } r-d \geqslant 0, \\ a & \text { when } r-d<0 .\end{cases}
$$

The suggested numerical scheme for 1.3 - 1.77 , which uses the upwind numerical flux function $F$ for the convection part and centered differencing for the parabolic part, takes the following predictor-corrector form for $j=0, \ldots, J_{L}-1$ and $n=N-1, \ldots, 0$ :

\section{Predictor step:}

$$
V_{j}^{n+1 / 2}=V_{j}^{n+1}+\lambda(r-d) x_{j} \Delta_{-} F\left(V_{j}^{n+1}, V_{j+1}^{n+1}\right)+\mu \frac{1}{2} \sigma^{2} x_{j}^{2} \Delta_{-} \Delta_{+} V_{j}^{n+1}-\Delta \operatorname{tr} V_{j}^{n+1} .
$$

\section{Corrector step:}

$$
V_{j}^{n}=V_{j}^{n+1 / 2}+\Delta t c\left(x_{j}\right) H\left(g\left(x_{j}\right)-V_{j}^{n+1 / 2}\right),
$$

We start the backward iteration 4.3 - 4.4 by setting

$$
V_{j}^{N}=g\left(x_{j}\right), \quad j=0, \ldots, J_{L} .
$$


We will impose the following Dirichlet condition at $x=0$ :

$$
V_{0}^{n+1 / 2}=V_{0}^{n}=g(0), \quad n=N-1, \ldots, 0 .
$$

Based on the asymptotic behavior of the American option value (1.1) as $x \uparrow \infty$, we will impose the following Dirichlet condition at $x=L$ :

$$
V_{J_{L}}^{n+1 / 2}=V_{J_{L}}^{n}=g(L), \quad n=N-1, \ldots, 0 .
$$

Note that when $V_{j}^{n+1 / 2} \leqslant g\left(x_{j}\right)$, the updating formula (4.4) reduces to

$V_{j}^{n}=V_{j}^{n+1}+\lambda(r-d) x_{j} \Delta_{-} F\left(V_{j}^{n+1}, V_{j+1}^{n+1}\right)+\mu \frac{1}{2} \sigma^{2} x_{j}^{2} \Delta_{-} \Delta_{+} V_{j}^{n+1}-\Delta t r V_{j}^{n+1}+\Delta t c\left(x_{j}\right)$.

Otherwise, 4.4) reduces to

$$
V_{j}^{n}=V_{j}^{n+1}+\lambda(r-d) x_{j} \Delta_{-} F\left(V_{j}^{n+1}, V_{j+1}^{n+1}\right)+\mu \frac{1}{2} \sigma^{2} x_{j}^{2} \Delta_{-} \Delta_{+} V_{j}^{n+1}-\Delta t r V_{j}^{n+1} .
$$

For the call (resp. put) option we have $c\left(x_{j}\right)=d x_{j}-r K\left(r K-d x_{j}\right)$ if $x_{j}>\frac{r}{d} K\left(\right.$ resp. $\left.<\frac{r}{d} K\right)$ ), and the updating formula (4.8) is possibly in effect. Otherwise, $c\left(x_{j}\right)=0$, and the updating formula 4.9 is effective.

We assume that the following parabolic CFL condition holds:

$$
\lambda|r-d| L+\mu \sigma^{2} L^{2}+\Delta t r \leqslant 1 .
$$

Note that when $\Delta(=\Delta x) \downarrow 0$, then also $\Delta t \downarrow 0$ by this condition.

The following lemma shows that the approximate solution $v_{\Delta}$ satisfies the early exercise constraint (1.8) and has (at most) linear growth as $x \uparrow \infty$, which implies that the finite difference scheme 4.4] is $L_{\text {loc }}^{\infty}$ stable.

Lemma 4.1 Suppose the parabolic CFL condition 4.10) holds. Then the predictor-corrector solution $\left\{V_{j}^{n}\right\}$ defined by (4.3) through (4.7) satisfies

$$
V_{j}^{n} \geqslant g\left(x_{j}\right), \quad j=0, \ldots, J_{L}, n=N, N-1, \ldots, 0 .
$$

Consequently, the approximate solution $v_{\Delta}$ defined by 4.2 and $\left\{V_{j}^{n}\right\}$ satisfies

$$
v_{\Delta}(t, x) \geqslant g(x)-O(\Delta x), \quad(t, x) \in \overline{Q_{T}^{L}} .
$$

Furthermore, there exist finite constants $C_{1}$ and $C_{2}$, independent of $\Delta$, such that

$$
V_{j}^{n} \leqslant C_{1}+C_{2} x_{j}, \quad j=0, \ldots, J_{L}, n=N, N-1, \ldots, 0 .
$$

For the call option, $C_{1}=0$ and $C_{2}=1$. For the put option, $C_{1}=K$ and $C_{2}=0$. Consequently,

$$
v_{\Delta}(t, x) \leqslant C_{1}+C_{2} x+O(\Delta x), \quad(t, x) \in \overline{Q_{T}^{L}} .
$$


Proof. The proof is inductive. Observe first that statements (4.11) and (4.13) hold for $(j=0$, $n=N, N-1, \ldots, 0),\left(j=J_{L}, n=N, N-1, \ldots, 0\right)$, and $\left(j=0, \ldots, J_{L}, n=N\right)$. For $\left(j=1, \ldots, J_{L}-1, n=N-1, \ldots, 0\right)$, we assume that a particular statement holds at time level $n+1$ and then seek to prove that it holds at time level $n$, starting with statement (4.11). First note that if $V_{j}^{n+1 / 2}>g\left(x_{j}\right)$, then

$$
V_{j}^{n}=V_{j}^{n+1 / 2}>g\left(x_{j}\right)
$$

and we are finished. In what follows, we therefore assume $V_{j}^{n+1 / 2} \leqslant g\left(x_{j}\right)$, so that

$$
V_{j}^{n}=V_{j}^{n+1 / 2}+\Delta t c\left(x_{j}\right)
$$

Introducing the function $\mathcal{S}$ defined by

$$
\begin{aligned}
\mathcal{S}\left(x_{j}, V_{j-1}^{n+1}, V_{j}^{n+1}, V_{j+1}^{n+1}\right)= & V_{j}^{n+1}+\lambda(r-d) x_{j} \Delta_{-} F\left(V_{j}^{n+1}, V_{j+1}^{n+1}\right) \\
& +\mu \frac{1}{2} \sigma^{2} x_{j}^{2} \Delta_{-} \Delta_{+} V_{j}^{n+1}-\Delta t r V_{j}^{n+1}
\end{aligned}
$$

we can write 4.3 as

$$
\mathcal{S}\left(x_{j}, V_{j-1}^{n+1}, V_{j}^{n+1}, V_{j+1}^{n+1}\right)-V_{j}^{n+1 / 2}=0 .
$$

Under the parabolic CFL condition (4.10), it is straightforward to check that

$$
\partial \mathcal{S} / \partial V_{j-1}^{n+1}, \partial \mathcal{S} / \partial V_{j}^{n+1}, \partial \mathcal{S} / \partial V_{j+1}^{n+1} \geqslant 0,
$$

which implies that the finite difference scheme is monotone. Set $g_{j}=g\left(x_{j}\right)$. Since by assumption $V_{j}^{n+1} \geqslant g_{j}$ for all $j$, we then have

$$
V_{j}^{n+1 / 2}=\mathcal{S}\left(x_{j}, V_{j-1}^{n+1}, V_{j}^{n+1}, V_{j+1}^{n+1}\right) \geqslant \mathcal{S}\left(x_{j}, g_{j-1}, g_{j}, g_{j+1}\right) \quad \forall j .
$$

Hence

$$
\begin{aligned}
V_{j}^{n+1 / 2} & \geqslant g_{j}+\lambda(r-d) x_{j} \Delta_{-} F\left(g_{j}, g_{j+1}\right)+\mu \frac{1}{2} \sigma^{2} x_{j}^{2} \Delta_{-} \Delta_{+} g_{j}-\Delta t r g_{j} \\
& \geqslant g_{j}+\lambda(r-d) x_{j} \Delta_{-} F\left(g_{j}, g_{j+1}\right)-\Delta t r g_{j} \quad \forall j
\end{aligned}
$$

where we have used the convexity of $g$ to derive the last inequality. To be concrete in what follows, we assume $r-d<0$, so that 4.17) reads

$$
V_{j}^{n+1 / 2} \geqslant g_{j}-\lambda(d-r) x_{j}\left(g_{j}-g_{j-1}\right)-\Delta t r g_{j}
$$

The case $r-d \geqslant 0$ can be treated similarly. We have two cases to consider:

(i) $x_{j} \leqslant K$. Then for the call option

$$
V_{j}^{n+1 / 2} \geqslant g_{j}-0 \geqslant g_{j}-\Delta t c\left(x_{j}\right),
$$

and for the put option

$$
V_{j}^{n+1 / 2} \geqslant g_{j}+\Delta t(d-r) x_{j}-\Delta t r\left(K-x_{j}\right)=g_{j}-\Delta t\left(r K-d x_{j}\right) \geqslant g_{j}-\Delta t c\left(x_{j}\right) .
$$


(ii) $x_{j}>K$. Then for the call option

$$
V_{j}^{n+1 / 2} \geqslant g_{j}-\Delta t(d-r) x_{j}-\Delta t r\left(x_{j}-K\right)=g_{j}-\Delta t\left(d x_{j}-r K\right) \geqslant g_{j}-\Delta t c\left(x_{j}\right) .
$$

and for the put option

$$
V_{j}^{n+1 / 2} \geqslant g_{j}-0 \geqslant g_{j}-\Delta t c\left(x_{j}\right)
$$

Summing up,

$$
V_{j}^{n+1 / 2} \geqslant g_{j}-\Delta t c\left(x_{j}\right) \quad \forall j .
$$

Plugging this lower bound for $V_{j}^{n+1 / 2}$ into 4.15 gives the desired result 4.11).

From (4.11) and the definition of $v_{\Delta}$ (see (4.2), we get 4.12).

Finally, we prove that (4.13) and (4.14) hold, starting with (4.13) and the put option. Assume that $V_{j}^{n+1} \leqslant K$ for all $j$. It then follows from from the monotonicity of $\mathcal{S}$ that

$$
V_{j}^{n+1 / 2} \leqslant K(1-\Delta t r)
$$

and hence, via (4.4),

$$
V_{j}^{n} \leqslant K(1-\Delta t r)+\Delta t c\left(x_{j}\right) \leqslant K \quad \forall j .
$$

For the call option, we assume $V_{j}^{n+1} \leqslant x_{j}$ for all $j$. Again from the monotonicity of $\mathcal{S}$, we get

$$
V_{j}^{n+1 / 2} \leqslant x_{j}(1-\Delta t r)-\Delta t(d-r) x_{j}=x_{j}-\Delta t d x_{j},
$$

and therefore

$$
V_{j}^{n} \leqslant x_{j}-\Delta t d x_{j}+c\left(x_{j}\right) \leqslant x_{j} \quad \forall j .
$$

This concludes the proof of 4.13, which also implies 4.14].

THEOREm 4.1 Suppose the parabolic CFL condition 4.10 holds. Denote by $v$ the unique viscosity solution of (1.3)-(1.7). Let $v_{\Delta}$ be the approximate solution defined by (4.2) and the predictor-corrector scheme 4.3 through 4.7 . Then

$$
v_{\Delta} \rightarrow v \quad \text { in } L_{\mathrm{loc}}^{\infty}\left(\overline{Q_{T}}\right) \text { as } \Delta \downarrow 0 .
$$

Proof. Let $\bar{v}$ and $\underline{v}$ be respectively the upper and lower weak limits of $v_{\Delta}$. We have $\bar{v} \in \operatorname{USC}\left(\overline{Q_{T}}\right)$, $\underline{v} \in \operatorname{LSC}\left(Q_{T}\right)$, and $\underline{v} \leqslant \bar{v}$ on $\overline{Q_{T}}$. In view of Lemma $4.1 \bar{v}, \underline{v}$ are finite at each point $(t, x) \in \overline{Q_{T}}$. More precisely, $0 \leqslant \bar{v}, \underline{v} \leqslant C(1+x)$ on $\overline{Q_{T}}$ for some constant $C>0$. For the moment, suppose that we can prove that $\bar{v}$ and $\underline{v}$ are respectively sub- and supersolutions of (1.3)-1.7]. An application of Theorem 2.1 then gives

$$
\bar{v} \leqslant \underline{v} \quad \text { on } \overline{Q_{T}} .
$$

We are thus finished since this implies $\bar{v}=\underline{v}$ on $\overline{Q_{T}}$ and, via Lemma 2.1 the approximate solution $v_{\Delta}$ converges as $\Delta \downarrow 0$ to the unique viscosity solution $v=\bar{v}=\underline{v}$ of (1.3)-(1.7).

We will first prove that $\bar{v}$ is a subsolution of (1.3). Pick a local maximizer $(t, x)$ of $\bar{v}-\phi$ for some $\phi \in C^{1,2}\left(\overline{Q_{T}}\right)$. Without loss of generality, we assume $\bar{v}(t, x)=\phi(t, x)$ and that the maximizer is strict. Moreover, we can assume $x>0$. In view of [4.1), there exists $\Delta x_{0}>0$ such that $(t, x)$ is an interior point of $Q_{T}^{L}$ for any $\Delta x<\Delta x_{0}$. In what follows, we assume $\Delta x<\Delta x_{0}$. In view of Lemma 4.1. we obviously have

$$
\bar{v}, \underline{v} \geqslant g \quad \text { on } \overline{Q_{T}} .
$$


First we consider the case $\bar{v}(t, x)=g(x)$. Since $\bar{v}(t, x)=g(x)$ and $\bar{v} \geqslant g$ on $\overline{Q_{T}}$, we conclude that $g-\phi$ has a local maximum at $(t, x)$. In [7], we proved that $g$ is a subsolution of (1.3). Hence (2.2) holds. Next we consider the case $\bar{v}(t, x) \geqslant g(x)+\delta$ for some $\delta>0$. Then $q^{*}(x, \bar{v}(t, x))=0$. By Lemma 2.1. there exist sequences $\left(t_{\Delta}, x_{\Delta}\right) \rightarrow(t, x)$ and $v_{\Delta}\left(t_{\Delta}, x_{\Delta}\right) \rightarrow \bar{v}(t, x)$ as $\Delta \downarrow 0$ such that $\left(t_{\Delta}, x_{\Delta}\right)$ is a local maximizer of $v_{\Delta}-\phi$ for each $\Delta x$. For any $\Delta x$ sufficiently small, we have $v_{\Delta}\left(t_{\Delta}, x_{\Delta}\right) \geqslant g\left(x_{\Delta}\right)+\delta / 2$. Without loss of generality, we can assume that there are integers $(j, n)$ such that $\left(t_{\Delta}, x_{\Delta}\right)=\left(t^{n}, x_{j}\right)$ and hence $v_{\Delta}\left(t_{\Delta}, x_{\Delta}\right)=V_{j}^{n}$. Note that

$$
V_{j}^{n}=V_{j}^{n+1 / 2}+\Delta t c\left(x_{j}\right) H\left(g\left(x_{j}\right)-V_{j}^{n+1 / 2}\right)
$$

where $V_{j}^{n+1 / 2}$ is defined in 4.3. It follows that

$$
V_{j}^{n+1 / 2}=V_{j}^{n}-\Delta t c\left(x_{j}\right) H\left(g\left(x_{j}\right)-V_{j}^{n+1 / 2}\right) \geqslant g\left(x_{j}\right)+\delta / 4,
$$

by choosing $\Delta$ sufficiently small. This implies that $V_{j}^{n}=V_{j}^{n+1 / 2}$, so that 4.4 reduces to

$$
V_{j}^{n}=V_{j}^{n+1}+\lambda(r-d) x_{j} \Delta_{-} F\left(V_{j}^{n+1}, V_{j+1}^{n+1}\right)+\mu \frac{1}{2} \sigma^{2} x_{j}^{2} \Delta_{-} \Delta_{+} V_{j}^{n+1}-\Delta t r V_{j}^{n+1} .
$$

Using the monotone function $\mathcal{S}$ defined in 4.16 , we can write 4.21 as

$$
\mathcal{S}\left(x_{j}, V_{j-1}^{n+1}, V_{j}^{n+1}, V_{j+1}^{n+1}\right)-V_{j}^{n}=0 .
$$

The fact that $v_{\Delta}-\phi$ has a maximum at $\left(t_{\Delta}, x_{\Delta}\right)$ implies

$$
\phi^{n+1} \geqslant V^{n+1}-\left(v_{\Delta}\left(t_{\Delta}, x_{\Delta}\right)-\phi\left(t_{\Delta}, x_{\Delta}\right)\right) .
$$

We have introduced the notation $\phi_{j}^{n}=\phi\left(t^{n}, x_{j}\right)$. By the monotonicity of $\mathcal{S}$ and 4.22 , we therefore obtain

$$
\begin{aligned}
\mathcal{S}\left(x_{j}, \phi_{j-1}^{n+1}, \phi_{j}^{n+1}\right. & \left., \phi_{j+1}^{n+1}\right)-\phi_{j}^{n} \\
& \geqslant \mathcal{S}\left(x_{j}, V_{j-1}^{n+1}, V_{j}^{n+1}, V_{j+1}^{n+1}\right)-V_{j}^{n}+\Delta \operatorname{tr}\left(v_{\Delta}\left(t_{\Delta}, x_{\Delta}\right)-\phi\left(t_{\Delta}, x_{\Delta}\right)\right) \\
& =\Delta \operatorname{tr}\left(v_{\Delta}\left(t_{\Delta}, x_{\Delta}\right)-\phi\left(t_{\Delta}, x_{\Delta}\right)\right) .
\end{aligned}
$$

Dividing (4.23) by $\Delta t$ and Taylor expanding around $\left(t_{\Delta}, x_{\Delta}\right)=\left(t^{n}, x_{j}\right)$ yields

$$
\partial_{t} \phi\left(t_{\Delta}, x_{\Delta}\right)+(r-d) x_{\Delta} \partial_{x} \phi\left(t_{\Delta}, x_{\Delta}\right)+\frac{1}{2} \sigma^{2} x_{\Delta}^{2} \partial_{x}^{2} \phi\left(t_{\Delta}, x_{\Delta}\right)-r v_{\Delta}\left(t_{\Delta}, x_{\Delta}\right) \geqslant-O(\Delta),
$$

where " $O(\Delta)$ " means " $\leqslant C(\Delta t+\Delta x)$ " for some constant $C \geqslant 0$ independent of $\Delta x$ and $\Delta t$. Letting $\Delta \downarrow 0$ in this inequality finishes the proof of the subsolution property. This shows that the finite difference scheme 4.21) is consistent.

We now prove that $\underline{v}$ is a supersolution of 1.3 . Pick a local minimizer $(t, x)$ of $\bar{v}-\phi$ with $\phi \in C^{1,2}\left(\overline{Q_{T}}\right)$. Without loss of generality, we can assume $\bar{v}(t, x)=\phi(t, x)$ and that the minimizer is strict. As before, we can find sequences $\left(t_{\Delta}, x_{\Delta}\right) \rightarrow(t, x)$ and $v_{\Delta}\left(t_{\Delta}, x_{\Delta}\right) \rightarrow \bar{v}(t, x)$ as $\Delta \downarrow 0$ such that $\left(t_{\Delta}, x_{\Delta}\right)$ is a local minimizer of $v_{\Delta}-\phi$ for each $\Delta$. We can also assume that each point 
$\left(t_{\Delta}, x_{\Delta}\right)$ coincides with a grid point. Since $\underline{v} \geqslant g$ on $\overline{Q_{T}}$, we have $q_{*}(x, \underline{v}(t, x))=0$. Note that (4.4) reads

$$
\mathcal{S}\left(x_{j}, V_{j-1}^{n+1}, V_{j}^{n+1}, V_{j+1}^{n+1}\right)-V_{j}^{n}=-\Delta t c\left(x_{j}\right) H\left(g\left(x_{j}\right)-V_{j}^{n+1 / 2}\right) \leqslant 0 .
$$

In view of this, arguing analogously to the subsolution case yields

$$
\partial_{t} \phi\left(t_{\Delta}, x_{\Delta}\right)+(r-d) x_{\Delta} \partial_{x} \phi\left(t_{\Delta}, x_{\Delta}\right)+\frac{1}{2} \sigma^{2} x_{\Delta}^{2} \partial_{x}^{2} \phi\left(t_{\Delta}, x_{\Delta}\right)-r v_{\Delta}\left(t_{\Delta}, x_{\Delta}\right) \leqslant O(\Delta) .
$$

Letting $\Delta \downarrow 0$ in this inequality finishes the proof of the supersolution property.

Finally, let us prove that $\bar{v}$ and $\underline{v}$ satisfy the terminal condition, i.e., $\left.\bar{v}\right|_{t=T} \leqslant g$ and $\left.\underline{v}\right|_{t=T} \geqslant g$ on $[0, \infty)$. In view of 4.20 , it is sufficient to prove that

$$
\left.\bar{v}\right|_{t=T} \leqslant g \quad \text { on }[0, \infty),
$$

which actually implies $\left.\bar{v}\right|_{t=T}=\underline{v}_{t=T}=g$ on $[0, \infty)$. Pick a function $\tilde{g} \in C^{2}([0, \infty))$ such that $\tilde{g} \geqslant g$ on $[0, \infty),\|\tilde{g}-g\|_{L^{\infty}([0, \infty))} \leqslant \delta$ for some $\delta>0$, and $\tilde{g}=g$ on $[\tilde{K}, \infty)$ with $\tilde{K}>\max (K,(r / d) K)$. Define $G=C(T-t)+\tilde{g}$ for a constant $C>0$, and note that $G>g$ on $Q_{T}$. Let $J_{\tilde{K}}$ be the integer such that $\tilde{K} \in I_{J_{\tilde{K}}}$. Set $G_{j}^{n}=G\left(t^{n}, x_{j}\right), \tilde{g}_{j}=\tilde{g}\left(x_{j}\right)$, and

$$
\tilde{C}=\max _{j=0, \ldots, J_{\tilde{K}}}\left\{(r-d) x_{j} \frac{1}{\Delta x} \Delta_{-} F\left(\tilde{g}_{j}, \tilde{g}_{j+1}\right)+\frac{1}{2} \sigma^{2} x_{j}^{2} \frac{1}{(\Delta x)^{2}} \Delta_{-} \Delta_{+} \tilde{g}_{j}-r \tilde{g}_{j}\right\} .
$$

Note that $\tilde{C}$ is finite and independent of $\Delta x$, since $\tilde{g} \in C^{2}$. Set $\tilde{c}=\max _{x \in[0, \tilde{K}+1]} c(x)$. For $j \leqslant J_{\tilde{K}}$, we have

$$
\mathcal{S}\left(x_{j}, G_{j-1}^{n+1}, G_{j}^{n+1}, G_{j+1}^{n+1}\right)-G_{j}^{n} \leqslant \Delta t\left[-C+\tilde{C}-c\left(x_{j}\right)+\tilde{c}\right] \leqslant-\Delta t c\left(x_{j}\right),
$$

provided we choose $C \geqslant \tilde{C}+\tilde{c}$. For $j>J_{\tilde{K}}$, it is easy to check that for any $C \geqslant 0$ we have

$$
\mathcal{S}\left(x_{j}, G_{j-1}^{n+1}, G_{j}^{n+1}, G_{j+1}^{n+1}\right)-G_{j}^{n}=\Delta t[-C-(d x-r K)] \leqslant-\Delta t c\left(x_{j}\right)
$$

for the call option and

$$
\mathcal{S}\left(x_{j}, G_{j-1}^{n+1}, G_{j}^{n+1}, G_{j+1}^{n+1}\right)-G_{j}^{n}=\Delta t[-C] \leqslant-\Delta t c\left(x_{j}\right)
$$

for the put option. Hence, by choosing $C \geqslant \tilde{C}+\tilde{c}$, we have

$$
\mathcal{S}\left(x_{j}, G_{j-1}^{n+1}, G_{j}^{n+1}, G_{j+1}^{n+1}\right)-G_{j}^{n} \leqslant-\Delta t c\left(x_{j}\right)
$$

for $j=1, \ldots, J_{L}-1$ and $n=N-1, \ldots, 0$. From the definition of the predictor-corrector scheme (4.3)-4.4), it follows that

$$
\mathcal{S}\left(x_{j}, V_{j-1}^{n+1}, V_{j}^{n+1}, V_{j+1}^{n+1}\right)-V_{j}^{n} \geqslant-\Delta t c\left(x_{j}\right)
$$

for $j=1, \ldots, J_{L}-1$ and $n=N-1, \ldots, 0$. We now claim that

$$
V_{j}^{n} \leqslant G_{j}^{n}, \quad j=0, \ldots, J_{L}, \quad n=N, N-1, \ldots, 0 .
$$


Obviously, by (4.5), (4.6), and (4.7), this is obviously true for $\left(j=0, \ldots, J_{L}, n=N\right),(j=$ $0, n=N, N-1, \ldots, 0)$, and $\left(j=J_{L}, n=N, N-1, \ldots, 0\right)$. To prove (4.28) for the remaining indices, we proceed by induction on $n$. Suppose $V_{j}^{n+1} \leqslant G_{j}^{n+1}$. Then, using 4.27, 4.26, and the monotonicity of $\mathcal{S}$, we reach the desired result

$$
\begin{aligned}
V_{j}^{n} & \leqslant \mathcal{S}\left(x_{j}, V_{j-1}^{n+1}, V_{j}^{n+1}, V_{j+1}^{n+1}\right)+\Delta t c\left(x_{j}\right) \\
& \leqslant \mathcal{S}\left(x_{j}, G_{j-1}^{n+1}, G_{j}^{n+1}, G_{j+1}^{n+1}\right)+\Delta t c\left(x_{j}\right)=G_{j}^{n} .
\end{aligned}
$$

From 4.28, it immediately follows that

$$
v_{\Delta}(t, x) \leqslant G(t, x)+O(\Delta x), \quad(t, x) \in \overline{Q_{T}^{L}},
$$

and therefore $\bar{v} \leqslant G$ on $\overline{Q_{T}}$. In particular, $\bar{v}(T, x) \leqslant \tilde{g}(x) \leqslant g(x)+\delta$. Since $\delta>0$ was arbitrary, the proof of 4.25 is finished.

REMARK Although this section shows that one can directly discretize the semilinear Black and Scholes equation (1.3) to obtain a "good" numerical scheme for computing the value of an American option, there is some numerical literature [12, 21, 25] on so-called penalty schemes. We claim that penalty schemes can be viewed as numerical schemes obtained by discretizing an approximation to the semilinear Black and Scholes equation (1.3), namely 3.1) with a suitable choice of $\varepsilon[12,25]$ or [3.5 with a suitable choice of $q_{\varepsilon}$. For example, the choice of $q_{\varepsilon}$ based on (3.7) and (3.11) is related to the penalty scheme used in [21]. The authors of [12, 21, 25] do not provide rigorous convergence proofs for their penalty schemes. At a semidiscrete level, we have already provided rather general convergence theorems (see Theorems 3.2, 3.3, and 3.1p. Regarding convergence proofs for numerical schemes based on discretizing (3.1) or (3.5), we only mention that the convergence arguments presented herein can be modified so as to apply to such numerical schemes.

From a computational point of view, the explicit predictor-corrector scheme 4.4 is not particularly useful because of the severe time step restriction imposed by the parabolic CFL condition (4.10). However, (4.10) can be easily avoided by replacing the explicit predictor step (4.3) by an implicit one. We end this section by briefly discussing an implicit predictor-corrector scheme. To simplify matters, we consider only the put option and set $d=0$. We then consider the following "CFL condition free" predictor-corrector scheme:

$$
\left\{\begin{array}{l}
V_{j}^{n+1 / 2}=V_{j}^{n+1}+\lambda r x_{j} \Delta_{-} V_{j+1}^{n+1 / 2}+\mu \frac{1}{2} \sigma^{2} x_{j}^{2} \Delta_{-} \Delta_{+} V_{j}^{n+1 / 2}-\Delta \operatorname{tr} V_{j}^{n+1 / 2}, \\
V_{j}^{n}=V_{j}^{n+1 / 2}+\Delta \operatorname{tr} K H\left(\left(K-x_{j}\right)^{+}-V_{j}^{n+1 / 2}\right) .
\end{array}\right.
$$

The terminal and boundary conditions for 4.29 ) are as before (see $4.57,(4.6)$, and 4.77$)$. Note that in 4.29 we do not have to solve a nonlinear algebraic system at each time step but merely a tridiagonal linear system. This makes 4.29 an efficient numerical scheme.

We have the following convergence theorem for 4.29 :

THEOREM 4.2 The statements in Lemma 4.1 and Theorem 4.1 hold true for the approximate solution $\left\{V_{j}^{n}\right\}$ generated by the implicit predictor-corrector scheme 4.29]. 
Proof. The proof follows very closely the proofs of Lemma 4.1 and Theorem 4.1 for the explicit predictor-corrector scheme, and hence we will omit most of it. Since we do not have any CFL condition relating the convergence to zero of $\Delta t$ with that of $\Delta x$, we need in this case to assume that $\Delta t$ goes to zero as a function of $\Delta x$ when $\Delta x \downarrow 0$. To illustrate a typical difference between the methods of proof for the explicit and implicit schemes, we will, however, prove that the early exercise constraint (4.11) holds. To this end, let us write the predictor step in 4.29$)$ as

$$
\frac{V_{j}^{n+1}-V_{j}^{n+1 / 2}}{\Delta t}+\mathcal{S}\left(x_{j}, V_{j-1}^{n+1 / 2}, V_{j}^{n+1 / 2}, V_{j+1}^{n+1 / 2}\right)=0,
$$

where

$$
\begin{aligned}
\mathcal{S}\left(x_{j}, V_{j-1}^{n+1 / 2}, V_{j}^{n+1 / 2}, V_{j+1}^{n+1 / 2}\right) & \\
\quad & r x_{j} \frac{1}{\Delta x} \Delta_{-} V_{j+1}^{n+1 / 2}+\frac{1}{2} \sigma^{2} x_{j}^{2} \frac{1}{(\Delta x)^{2}} \Delta_{-} \Delta_{+} V_{j}^{n+1 / 2}-r V_{j}^{n+1 / 2} .
\end{aligned}
$$

Observe that

$$
\partial \mathcal{S} / \partial V_{j-1}^{n+1 / 2}, \partial \mathcal{S} / \partial V_{j+1}^{n+1 / 2} \geqslant 0 .
$$

For any $j$, set $g_{j}=g\left(x_{j}\right)$ and $W_{j}^{n+p}=V_{j}^{n+p}-g_{j}$ with $p=1,1 / 2$. Observe that $W_{j}^{n+1} \geqslant 0$ for all $j$. The scheme for $W_{j}^{n+1 / 2}$ reads

$$
\frac{W_{j}^{n+1}-W_{j}^{n+1 / 2}}{\Delta t}+\mathcal{S}\left(x_{j}, W_{j-1}^{n+1 / 2}, W_{j}^{n+1 / 2}, W_{j+1}^{n+1 / 2}\right)+\mathcal{S}\left(x_{j}, g_{j-1}, g_{j}, g_{j+1}\right)=0 .
$$

Let $W_{\ell}^{n+1 / 2}=\min _{j} W_{j}^{n+1 / 2}$ for some $\ell$ and assume $W_{\ell}^{n+1 / 2}<0$ (otherwise there is nothing to prove). Then

$$
\frac{W_{\ell}^{n+1}-W_{\ell}^{n+1 / 2}}{\Delta t}+\mathcal{S}\left(x_{\ell}, W_{\ell}^{n+1 / 2}, W_{\ell}^{n+1 / 2}, W_{\ell}^{n+1 / 2}\right)+\mathcal{S}\left(x_{\ell}, g_{\ell-1}, g_{\ell}, g_{\ell+1}\right) \leqslant 0,
$$

which implies that

$$
(1+\Delta t r) W_{\ell}^{n+1 / 2} \geqslant \Delta t \mathcal{S}\left(x_{\ell}, g_{\ell-1}, g_{\ell}, g_{\ell+1}\right) \geqslant-\Delta t r K,
$$

where the last inequality follows as in the proof of (4.11) for the explicit predictor-corrector scheme. In particular, $W_{j}^{n+1 / 2} \geqslant-\Delta \operatorname{tr} K$ for all $j$. Plugging this into 4.29) yields 4.11, and hence 4.12).

Similarly, we can prove that (4.13) and (4.14) hold.

By the monotonicity of $\mathcal{S}$ in two of the variables, the proof that Theorem 4.1 holds for (4.29) is more or less identical to the proof for the explicit predictor-corrector scheme.

\section{The Brennan and Schwartz algorithm}

By applying a predictor-corrector discretization to 3.1 , we can devise a numerical scheme that is identical to the one analyzed in Section 4 except that the corrector step (4.4) is replaced by the following one:

$$
V_{j}^{n}=V_{j}^{n+1 / 2}+\frac{\Delta t}{\varepsilon}\left(g\left(x_{j}\right)-V_{j}^{n+1 / 2}\right)^{+},
$$


where $\varepsilon$ has to be chosen. We are here interested in the particular choice $\varepsilon=\Delta t$, which results in the following explicit scheme:

$$
\left\{\begin{aligned}
& V_{j}^{n+1 / 2}= V_{j}^{n+1}+\lambda(r-d) x_{j} \Delta_{-} F\left(V_{j}^{n+1}, V_{j+1}^{n+1}\right) \\
&+\mu \frac{1}{2} \sigma^{2} x_{j}^{2} \Delta_{-} \Delta_{+} V_{j}^{n+1}-\Delta t r V_{j}^{n+1}, \\
& V_{j}^{n}=\max \left(V_{j}^{n+1 / 2}, g\left(x_{j}\right)\right) .
\end{aligned}\right.
$$

The terminal and boundary conditions for (5.1) are as in Section 4 (see (4.5), (4.6), and (4.7)). We call 5.1) the Brennan and Schwartz algorithm, referring to the classical scheme studied by Brennan and Schwartz in [8]. The theoretical justification of the Brennan and Schwartz algorithm is a delicate issue that has been treated by Jaillet, Lamberton, and Lapeyre [15, 16] using the theory of quasi-variational inequalities due to Bensoussan and Lions [5, 6]. Viscosity solution theory for quasi-variational inequalities can be found in [22, 23]. The purpose of this section is to show that we can use the mathematical framework for the semilinear Black and Scholes equation to give an elementary convergence proof for the Brennan and Schwartz scheme defined in (5.1).

We have the following theorem:

THEOREM 5.1 Suppose the parabolic CFL condition 4.10 holds. Then the statements in Lemma 4.1 and Theorem 4.1 hold true for the approximate solution $\left\{V_{j}^{n}\right\}$ generated by the Brennan and Schwartz algorithm (5.1).

Proof. The proof follows the lines of the proofs of Lemma 4.1 and Theorem 4.1. and hence we will be rather brief here. We trivially have $V_{j}^{n} \geqslant g_{j}$ for all $j, n$, so that 4.11) and hence (4.12) hold. The upper bounds (4.13) and (4.14) can be proved as before. Let us now prove that the upper weak limit $\bar{v}$ is a subsolution of (1.3)-(1.7), starting with the terminal condition (1.7). Since $V_{j}^{n+1} \geqslant g_{j}$ for all $j$, we know from 4.18 that

$$
-\left(g\left(x_{j}\right)-V_{j}^{n+1 / 2}\right)^{+} \geqslant-\Delta t c\left(x_{j}\right) \quad \forall j .
$$

Plugging this into [5.1, we get

$$
\mathcal{S}\left(x_{j}, V_{j-1}^{n+1}, V_{j}^{n+1}, V_{j+1}^{n+1}\right)-V_{j}^{n} \geqslant-\Delta t c\left(x_{j}\right) \quad \forall j
$$

where $\mathcal{S}$ is defined in 4.16. Now proceeding exactly as in the proof of Theorem 4.1, we end up with (4.25). It remains to prove that $\bar{v}$ is a subsolution of (1.3). Pick a strict local maximizer $(t, x)$ of $\bar{v}-\phi$ for some $\phi \in C^{1,2}\left(Q_{T}\right)$ with $x>0$. The case $\bar{v}(t, x)=g(x)$ can treated as in the proof of Theorem 4.1 Let us therefore assume $\bar{v}(t, x)>g(x)+\delta$ for some $\delta>0$. Then $q^{*}(x, \bar{v}(t, x))=0$. By Lemma 2.1. there exist sequences $\left(t_{\Delta}, x_{\Delta}\right) \rightarrow(t, x)$ and $v_{\Delta}\left(t_{\Delta}, x_{\Delta}\right) \rightarrow \bar{v}(t, x)$ as $\Delta \downarrow 0$ such that $\left(t_{\Delta}, x_{\Delta}\right)$ is a local maximizer of $v_{\Delta}-\phi$ for each $\Delta x$. For any $\Delta x$ sufficiently small, we have $v_{\Delta}\left(t_{\Delta}, x_{\Delta}\right)>g\left(x_{\Delta}\right)$. Pick integers $(j, n)$ such that $\left(t_{\Delta}, x_{\Delta}\right)=\left(t^{n}, x_{j}\right)$ and $v_{\Delta}\left(t_{\Delta}, x_{\Delta}\right)=V_{j}^{n}$. Using the monotone function $\mathcal{S}$ defined in 4.16, we can write (5.1) as

$$
\mathcal{S}\left(x_{j}, V_{j-1}^{n+1}, V_{j}^{n+1}, V_{j+1}^{n+1}\right)-V_{j}^{n}=0 .
$$

We can now conclude exactly as in the proof of Theorem 4.1 . 
Let us now prove that the lower weak limit $\underline{v}$ is a supersolution of (1.3)-(1.7). In view of 4.20), we already know that $\underline{v}$ satisfies the terminal condition. Upon replacing 4.24 by

$$
\mathcal{S}\left(x_{j}, V_{j-1}^{n+1}, V_{j}^{n+1}, V_{j+1}^{n+1}\right)-V_{j}^{n}=-\left(g\left(x_{j}\right)-V_{j}^{n+1 / 2}\right)^{+} \leqslant 0,
$$

the proof that $\underline{v}$ is a supersolution of $(1.3)$ goes exactly as in the proof of Theorem 4.1 .

\section{A numerical example}

In this section, we test the predictor-corrector scheme defined and analyzed in Section 4 . In the computer program that has been implemented, we specify the spatial discretization parameter $\Delta x$ and then choose $\Delta t$ according to

$$
\Delta t \leqslant \frac{(\Delta x)^{2}}{\Delta x|r-d| L+\sigma^{2} L^{2}+(\Delta x)^{2} r},
$$

so that the parabolic CFL condition (4.10) holds. We present here only numerical results for the put option with $d=0$. Furthermore, we use the following parameters:

$$
r=0.1, \quad \sigma=0.2, \quad K=1, \quad T=1.0, \quad L=4, \quad \Delta x=0.00533, \quad \Delta t=0.0043 .
$$

The choice $\Delta x=0.00533$ corresponds to 75 grid points and $\Delta t=0.0043$ is chosen according to 6.1. For comparison, we use an "exact solution" computed by the predictor-corrector scheme on a very fine grid. The exact and numerical solutions are displayed in Figure 1 . The predictor-corrector scheme gives a fairly good approximation to the exact solution. The largest difference is seen in the early exercise region (which roughly speaking corresponds to " $x>0.8-0.9$ "). However, this difference is largely due to the use of a first order upwind discretization of the convection term in the Black and Scholes operator. By using a high order discretization of the convection term, this difference can be greatly reduced.

For further comparison, we have also computed a solution with the Brennan and Schwartz algorithm (5.1). The same set of discretization parameters have been used and the result is shown in Figure 2 In the "visual norm" the predictor-corrector and Brennan and Schwartz schemes produce solutions of more or less the same quality. This is also confirmed by looking at "zoom-in plots" (like those in Figures 4 comparing the predictor-corrector and penalty schemes). We do not present these plots here, since one can hardly see any difference between the two schemes.

As mentioned before, instead of discretizing the semilinear Black and Scholes equation (1.3), one can discretize the approximate semilinear Black and Scholes equation (3.5). To be concrete, consider (3.5) with $q_{\varepsilon}$ defined via (3.7) and $H_{\varepsilon}(\xi)=\varepsilon /(\varepsilon-\xi)$. An explicit finite difference discretization (3.5) with this choice of $q_{\varepsilon}$ produces the following penalty scheme:

$$
\begin{aligned}
V_{j}^{n}= & V_{j}^{n+1}+\lambda(r-d) x_{j} \Delta_{-} V_{j+1}^{n+1}+\mu \frac{1}{2} \sigma^{2} x_{j}^{2} \Delta_{-} \Delta_{+} V_{j}^{n+1} \\
& -\Delta \operatorname{tr} V_{j}^{n+1}+\Delta \operatorname{tr} K \frac{\varepsilon}{\varepsilon+V_{j}^{n+1}-\left(K-x_{j}\right)}
\end{aligned}
$$

where $\varepsilon>0$ is a parameter that has to be specified. The terminal and boundary conditions for (6.2) are as in Section 4 We note that the penalty scheme (6.2) coincides with the one used in [21]. 


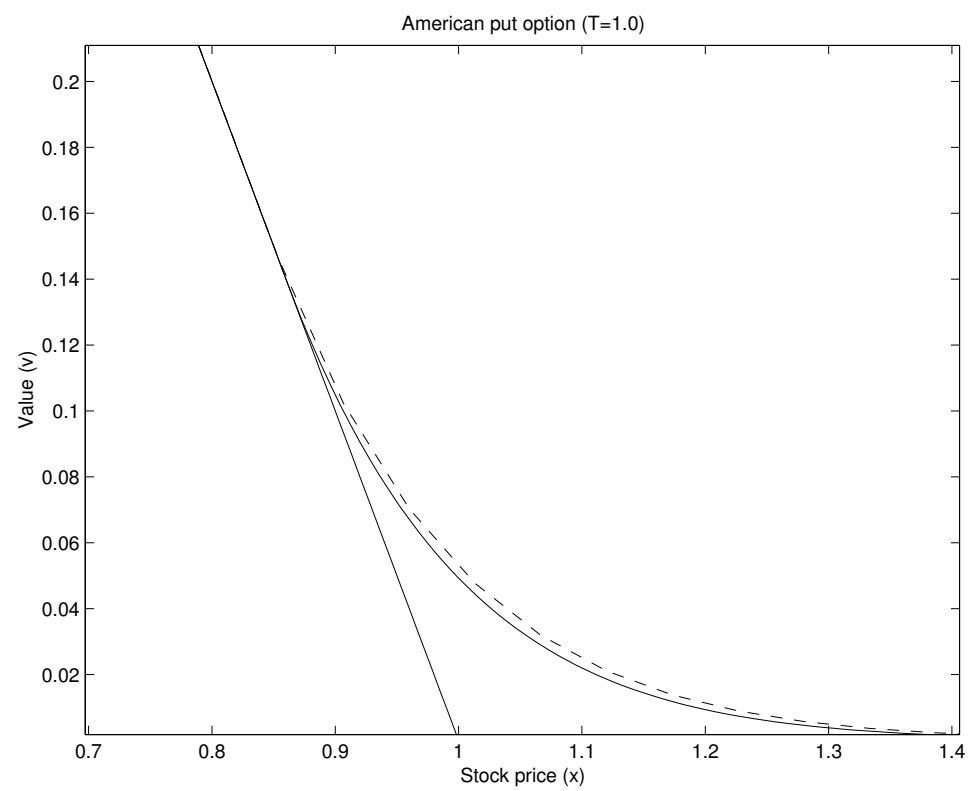

FIG. 1. The price of the American put option with expiration time $T=1.0$ : the exact solution along with the payoff function (solid line) and the predictor-corrector solution (dashed line)

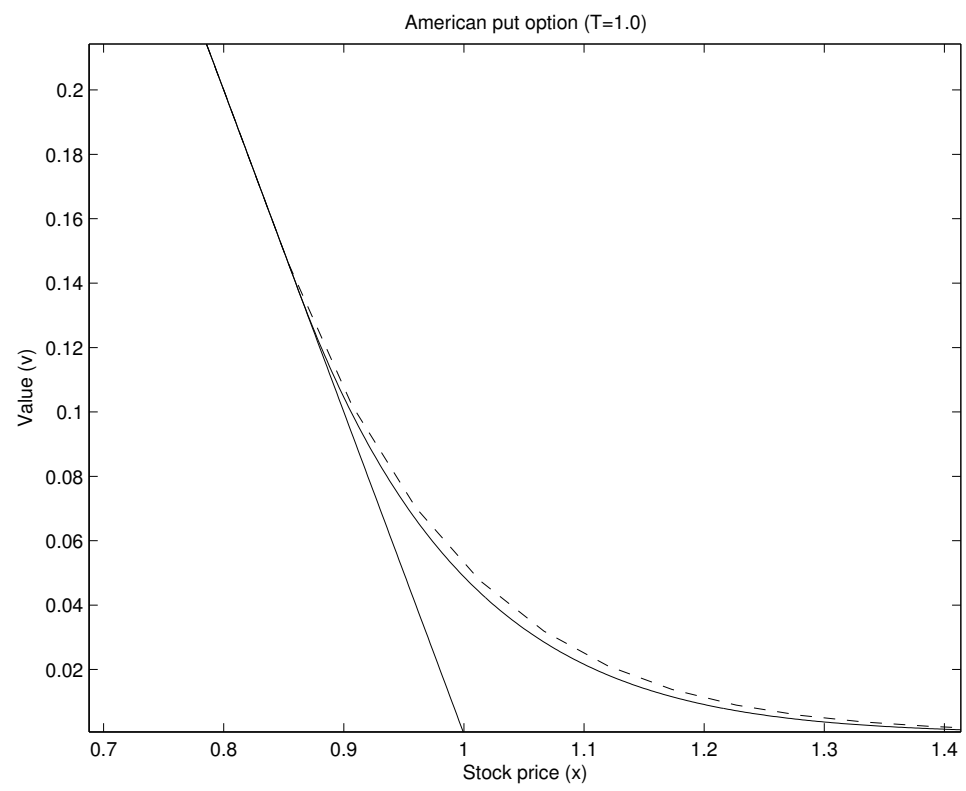

FIG. 2. The price of the American put option with expiration time $T=1.0$ : the exact solution along with the payoff function (solid line) and the Brennan and Schwartz solution (dashed line) 


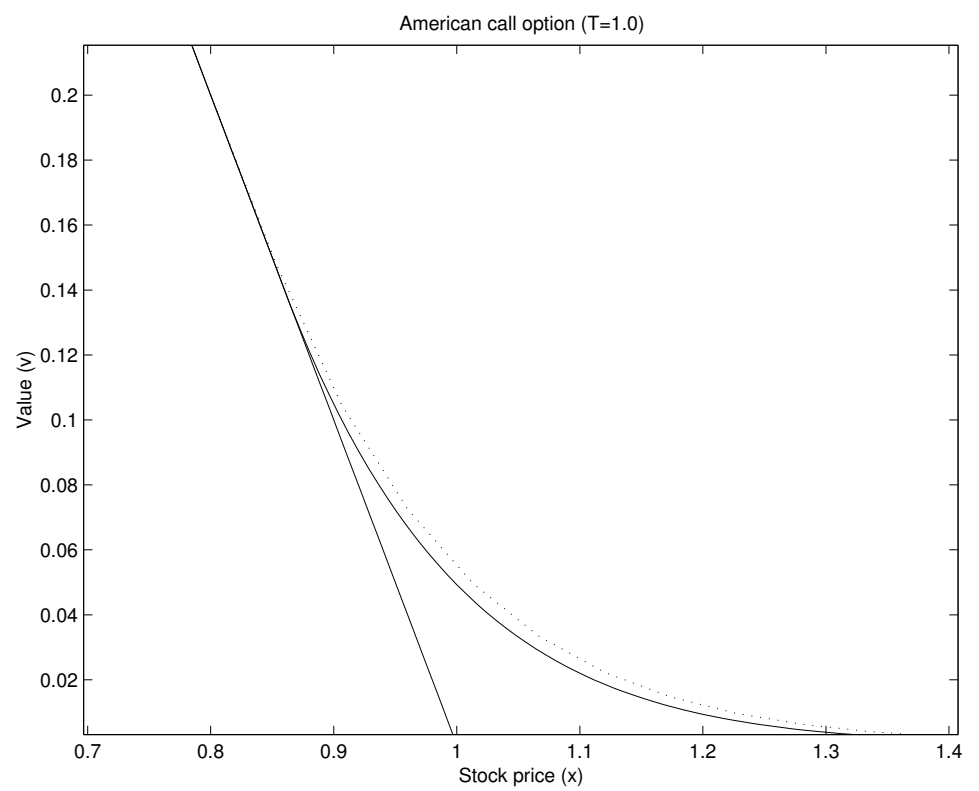

FIG. 3. The price of the American put option with expiration time $T=1.0$ : the exact solution along with the payoff function (solid line) and the penalty solution (dashed line)

In [21], it was proved that (6.2) satisfied a discrete analog of the early exercise constraint (1.8) provided the following strengthened CFL condition holds:

$$
\Delta t \leqslant \frac{(\Delta x)^{2}}{\Delta x r L+\sigma^{2} L^{2}+r(\Delta x)^{2}+\frac{r K}{\varepsilon}(\Delta x)^{2}} .
$$

Adopting the method of proof herein, one can also prove under this CFL condition that the approximate solutions generated by the penalty scheme 6.2 converge to the unique viscosity solution of (1.3)-(1.7) (i.e., the American put option value).

For the numerical example, the penalty scheme used the same discretization parameters as the predictor-corrector scheme. This forces us to choose $\varepsilon=0.0009$. The exact and numerical solutions are displayed in Figure 3 . The penalty solution is comparable to the predictor-corrector solution. However, the "zoom-in plots" in Figure 4 reveal that the penalty solution consistently lies above the predictor-corrector solution. This is not surprising since the reaction term in 6.2 is nonzero (but small) also in the optimal exercise region. Although we did not gain anything here by using a continuous approximation $q_{\varepsilon}$ instead of the discontinuous nonlinear reaction term $q$ in $(1.4)$, it may be an advantage to use $q_{\varepsilon}$ instead of $q$ in a numerical scheme in which the nonlinear reaction term is going to be discretized implicitly. But this remains to be investigated.

\section{Acknowledgments}

F. E. Benth is partially supported by MaPhySto, which is funded by a research grant from the Danish National Research Foundation. A part of this work was done while K. H. Karlsen and K. Reikvam 

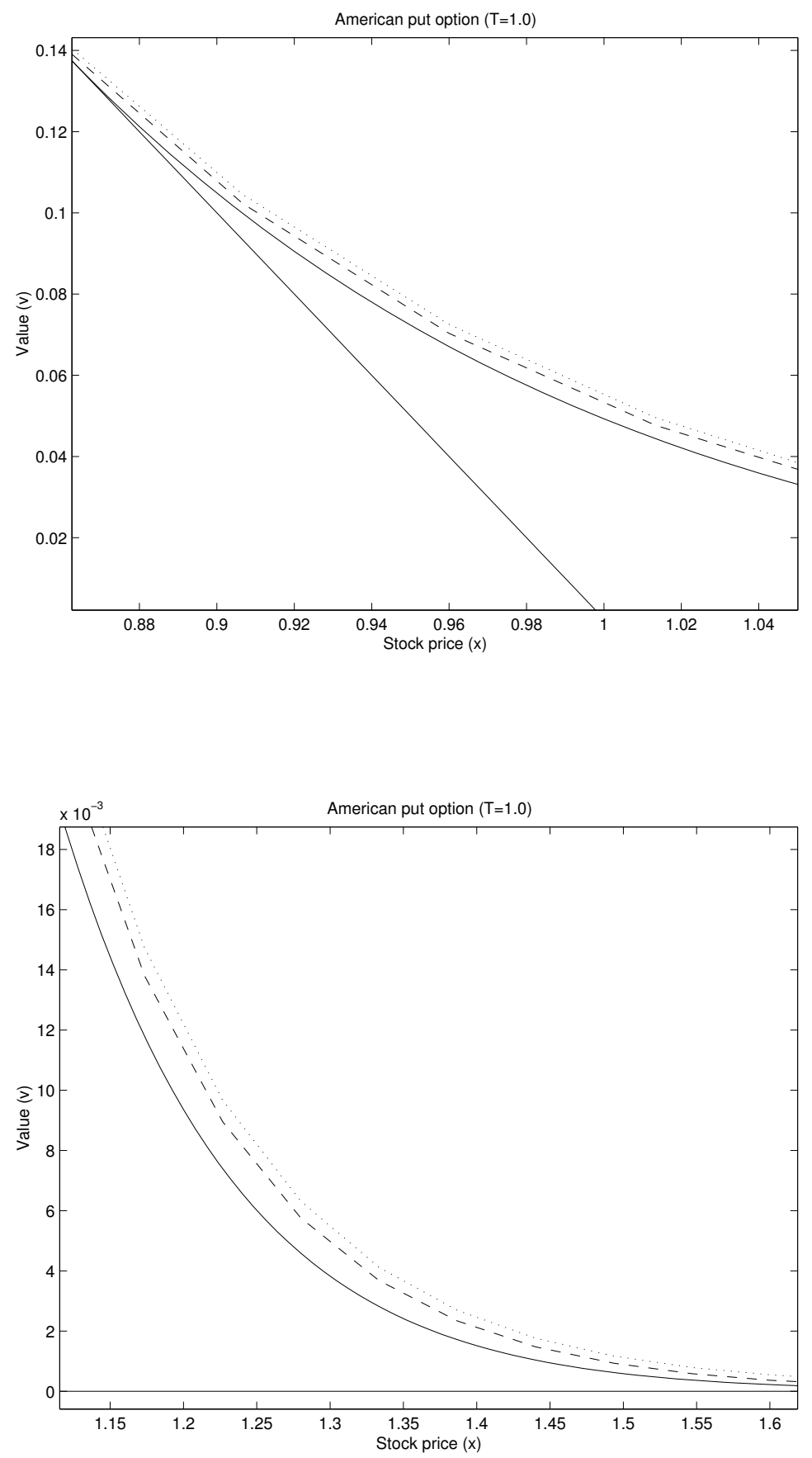

FIG. 4. "Zoom-in plots" of the exact solution (solid line), the predictor-corrector solution (dashed line), and the penalty solution (dotted line). 
were visiting the Department of Mathematics, and K. H. Karlsen also the Institute for Pure and Applied Mathematics (IPAM), at the University of California, Los Angeles (UCLA). K. H. Karlsen is grateful to IPAM and the project Nonlinear partial differential equations of evolution typetheory and numerics, which is part of the BeMatA program of The Research Council of Norway, for financial support.

\section{REFERENCES}

1. BARLES, G. Convergence of numerical schemes for degenerate parabolic equations arising in finance theory. Numerical Methods in Finance, Cambridge Univ. Press, Cambridge (1997), 1-21. Zbl 0898.90015 MR 1470506

2. Barles, G., DAher, C., \& Romano, M. Convergence of numerical schemes for parabolic equations arising in finance theory. Math. Models Methods Appl. Sci. 5 (1995), 125-143. Zbl 0822.65056 MR 1315000

3. Barles, G. \& Perthame, B. Comparison principle for Dirichlet-type Hamilton-Jacobi equations and singular perturbations of degenerated elliptic equations. Appl. Math. Optim. 21 (1990), 21-44. Zbl 0691.49028 MR 1014943

4. Barles, G. \& Souganidis, P. E. Convergence of approximation schemes for fully nonlinear second order equations. Asymptotic Anal. 4 (1991), 271-283. Zbl 0729.65077 MR 1115933

5. Bensoussan, A. \& Lions, J.-L. Applications of Variational Inequalities in Stochastic Control. NorthHolland, Amsterdam (1982). Zbl 0478.49002 MR 0653144

6. Bensouss An, A. \& LiOns, J.-L. Impulse Control and Quasivariational Inequalities. Gauthier-Villars, Montrouge (1984). MR 0756234

7. Benth, F. E., Karlsen, K. H., \& Reikvam, K. A semilinear Black and Scholes partial differential equation for valuing American options Finance Stoch. 7 (2003), 277-298. MR 1994910

8. Brennan, M. J. \& SChwartz, E. S. The valuation of American put options. J. Finance 32 (1977), 449-462.

9. Crandall, M. G., Ishit, H., \& LiOnS, P.-L. User's guide to viscosity solutions of second order partial differential equations. Bull. Amer. Math. Soc. (N.S.) 27 (1992), 1-67. Zbl 0755.35015 MR 1118699

10. DuffIE, D. Dynamic Asset Pricing Theory. Princeton Univ. Press, Princeton (1996). Zbl pre01869272

11. Fleming, W. H. \& Soner, H. M. Controlled Markov Processes and Viscosity Solutions. Springer, New York (1993). Zbl 0773.60070 MR 1199811

12. Forsyth, P. A. \& VetZal, K. R. Quadratic convergence for valuing American options using a penalty method. SIAM J. Comput. Sci. 23 (2002), 2095-2122. MR 1923727

13. IsHII, H. Hamilton-Jacobi equations with discontinuous Hamiltonians on arbitrary open sets. Bull. Fac. Sci. Engrg. Chuo Univ. 28 (1985), 33-77. Zbl 0937.35505 MR 0845397

14. IsHII, H. A boundary value problem of the Dirichlet type for Hamilton-Jacobi equations. Ann. Scuola Norm. Sup. Pisa Cl. Sci. (4) 16 (1989), 105-135. Zbl 0701.35052 MR 1056130

15. Jaillet, P., LAmberton, D., \& LAPEyre, B. Inéquations variationnelles et théorie des options. C. R. Acad. Sci. Paris Sér. I Math. 307 (1988), 961-965. Zbl 0825.90044 MR 0977168

16. Jaillet, P., Lamberton, D., \& Lapeyre, B. Variational inequalities and the pricing of American options. Acta Appl. Math. 21 (1990), 263-289. Zbl 0714.90004 MR 1096582

17. Karatzas, I. \& Shreve, S. E. Methods of Mathematical Finance. Springer, New York (1998). Zbl 0941.91032 MR 1640352

18. Kholodnyi, V. A. A nonlinear partial differential equation for American options in the entire domain of the state variable. Nonlinear Anal. 30 (1997), 5059-5070. Zbl 0911.90029 MR 1726008

19. Musiela, M. \& RutKowski, M. Martingale Methods in Financial Modelling. Springer, Berlin (1997). Zbl 0906.60001 MR 1474500 
20. Myneni, R. The pricing of the American option. Ann. Appl. Probab. 2 (1992), 1-23. Zbl 0753.60040 MR 1143390

21. Nielsen, B. F., Skavhaug, O., \& Tveito, A. Penalty and front-fixing methods for the numerical solution of American option problems. J. Comp. Finance 5 (2002), 69-97.

22. ØKSEndal, B. \& ReikVAM, K. Viscosity solutions of optimal stopping problems. Stoch. Stoch. Rep. 62 (1998), 285-301. Zbl 0913.60037| MR 1613260

23. Pham, H. Optimal stopping of controlled jump diffusion processes: a viscosity solution approach. J. Math. Systems Estim. Control 8 (1998), 27 pp. (electronic). Zbl 0899.60039 MR 1650147

24. ShIRYAYEV, A. N. Optimal Stopping Rules. Springer, New York (1978). Zbl 0391.60002 MR 0468067

25. ZVAn, R., Forsyth, P. A., \& Vetzal, K. R. Penalty methods for American options with stochastic volatility. J. Comput. Appl. Math. 91 (1998), 199-218. Zbl 0945.65005 MR 1628686 